Thomas Gloning

\title{
Spielarten von Kontroversen in der Wissenschaftskommunikation des 16. bis 18. Jahrhunderts
}

\section{Kontroversen in der historischen Wissenschaftskommunikation}

Kontroversen sind in der Frühen Neuzeit wichtige Bestandteile der „historischen Wissenschaftssprache“ bzw. genauer: des wissenschaftlichen Sprachgebrauchs oder der Wissenschaftskommunikation. Denn auch wenn Beiträge zu Kontroversen typische lexikalische und syntaktische Besonderheiten aufweisen, so sind diese doch eingebettet in eine mehr oder weniger stabile Praxis der gelehrten Auseinandersetzung, die in einem historisch-pragmatischen Beschreibungsrahmen bearbeitet werden muss. Solche Formen der Auseinandersetzung betreffen im 16. bis 18. Jahrhundert - um diesen Zeitraum soll es hier gehen - ganz unterschiedliche Disziplinen, z. B. die Medizin, die damals noch näher beieinander liegenden Bereiche der Astronomie und der Astrologie, die Theologie mit ihren Ausläufern in ganz handfeste Bereiche der Religionspolitik, die Alchemie, Metallurgie und die daraus erwachsende moderne Chemie, die Philosophie und weitere ,wissenschaftsnahe“ fachliche Felder, z. B. im Bereich der Technik.

Solche fachlichen Auseinandersetzungen konnten sich zum Beispiel in den gelehrten Briefwechseln entfalten, sie hatten dann einen stärker privaten Charakter bzw. einen geringen Öffentlichkeitsgrad, der sich auf die Zirkel beschränkte, in denen die Briefe ausgetauscht wurden. Mit gedruckten Streitschriften konnte man eine breitere fachliche Öffentlichkeit erreichen, bei manchen Themen etwa im Spannungsfeld von Theologie und Religionspolitik sicher auch eine erweiterte Öffentlichkeit. Kontroversen waren in solchen Fällen in die

\footnotetext{
Anmerkung: Für hilfreiche Hinweise und Unterstützung danke ich Roman Degreif, Gerd Fritz, Dennis Kaltwasser, Heike Müller und Andre Pietsch. Ich danke auch den beiden anonymen GutachterInnen des Bandes: für die Anerkennung, die genaue Lektüre und die nützlichen Hinweise.
}

Thomas Gloning, Justus-Liebig-Universität Gießen, Institut für Germanistik, Otto-BehaghelStr. 10 B, 35394 Gießen, E-Mail: thomas.gloning@germanistik.uni-giessen.de

Ә Open Access. (c) 2018 Thomas Gloning, publiziert von De Gruyter. (c) BY-NC-ND Dieses Werk ist lizenziert unter der Creative Commons Attribution-NonCommercial-NoDerivatives 4.0 Lizenz. https://doi.org/10.1515/9783110476958-005 
medialen, institutionellen und wirtschaftlichen Zusammenhänge des Buchdrucksystems eingebunden, seit dem späten 17 . Jahrhundert kamen die wissenschaftlichen Zeitschriften auf, die dann gravierende Veränderungen für die Kontroversenpraxis hervorbrachten.

Wissenschaftliche Kontroversen der Frühen Neuzeit kann man demnach als eine Familie von medial und institutionell eingebetteten „kommunikativen Gattungen“ (Th. Luckmann) ansehen, die sich evolutionär herausgebildet haben (u. a. mit Wurzeln in der Disputationspraxis) ${ }^{1}$ und die neben Aspekten einer weitreichenden Schemabildung aber auch nicht geringe Realisierungsspielräume in den unterschiedlichen fachlichen Bereichen und im Hinblick auf individuelle Profile aufweisen können.

$\mathrm{Zu}$ den Aspekten der Außenstruktur der Kontroversen gehören, wie bereits erwähnt, die institutionelle Einbettung dieser Praxis an den Universitäten und ihren Fakultäten, an den Höfen, die in der Frühen Neuzeit zum Teil bedeutende Wissenschaftszentralen waren, man denke an den Hof Rudolfs II. in Prag um 1600, aber auch in religiösen Institutionen, zum Beispiel im Jesuitenorden, der über die Theologie hinaus auch exzellente und streitbare Wissenschaftler in anderen fachlichen Bereichen hervorgebracht hat. Die beiden großen medialen Infrastrukturen für die Kontroversenpraxis waren der Buchdruck und die gelehrten Briefwechsel.

Die Beschreibung der Aspekte der Binnenstrukturierung von Kontroversen, ihrer Spielarten und ihrer historischen Entwicklung im Längsschnitt sind die zentrale Aufgabe, die im Rahmen einer historischen Pragmatik der Wissenschaftskommunikation bearbeitet werden muss. Auf diese Aufgabe lassen sich folgende Leitfragen und Teilaufgaben beziehen:

1. Welche Funktionen haben Kontroversen im gelehrten „Stoffwechsel“ der Wissenschaft in der Frühen Neuzeit? Was leisten dabei einzelne Kontroversenbeiträge?

2. Welche fachlich orientierten Texttypen und welche Darstellungsformen wurden im Rahmen von Wissenschaftskontroversen in der Frühen Neuzeit verwendet? Wie kann man ihre typische Organisation, aber auch ihre Realisierungsspielräume charakterisieren? Wie hat sich das Repertoire der Darstellungsformen historisch entwickelt? Wie haben sich Aspekte der sprachlich-pragmatischen Organisation von Darstellungsformen im Rahmen von Kontroversen historisch entwickelt?

$1 \mathrm{Zu}$ den Spielarten und zur Geschichte der Disputation vgl. u. a. Gindhart \& Kundert 2010; Gindhart, Marti \& Seidel 2016; Sdzuj, Seidel \& Zegowitz 2012. Zum Zusammenhang des „Disputationsmodells“ mit Streitschriften-Kontroversen vgl. Fritz 2017, Kap. 10, insbesondere Abschnitt 10.2. 
Im folgenden Abschnitt charakterisiere ich zunächst einen historisch-pragmatischen Beschreibungs- und Analyserahmen für diese Aufgabe. In den darauf folgenden Abschnitten erläutere ich diesen theoretisch-methodischen Rahmen anhand ausgewählter wissenschaftlicher Kontroversen des 16. bis 18. Jahrhunderts. Dabei sollen grundlegende Aspekte der Organisation historischer Wissenschaftskontroversen aus dieser Zeit, aber auch Realisierungsspielräume und historische Entwicklungstendenzen deutlich werden.

\section{Die pragmatische Form historischer Wissenschaftskontroversen: ein Beschreibungs- und Analyserahmen}

Die pragmatische Form historischer Wissenschaftskontroversen kann man in einem funktionalen, handlungstheoretischen Rahmen analysieren und beschreiben (vgl. Fritz 2010; Gloning 1999). Zentral sind dabei eine Reihe von Grundannahmen, die sich folgendermaßen formulieren lassen. Der kommunikative Gebrauch von Texten ist eine Form des komplexen sprachlichen Handelns. Texte sind Instrumente des sprachlichen Handelns, sie dienen jeweils spezifischen kommunikativen Funktionen. Im Lauf der Kommunikationsgeschichte haben sich Muster des Handelns mit Texten, historische Texttypen, herausgebildet, die auf wiederkehrende Funktionen gerichtet sind. Dabei spielen sich Prozesse der Verfestigung nach einem Invisible-hand-Muster ab, bei denen das individuelle Handeln vieler Sprecher bzw. Schreiber, wenn es gleichen oder ähnlichen Zielen folgt, überindividuelle Resultate der Verfestigung hervorbringt (Beckmann \& König 1995; Keller 1990). Einer Idee von Thomas Luckmann (1986) folgend, kann man das Repertoire der auf diese Weise abgedeckten kommunikativen Funktionen und der darauf bezogenen Formen der Kommunikation als einen kommunikativen „Haushalt“ betrachten.

Die historische Ausprägung einzelner Texttypen oder auch komplexer Kommunikationsformen wie z. B. Kontroversen kann man auf das Zusammenspiel grundlegender pragmatischer Parameter zurückführen, die ich im Folgenden kurz erläutere.

(1) Ein übergeordneter Aspekt ist die Frage, welche Funktion(en) ein Text bzw. eine komplexe Kommunikationsform, in die der Gebrauch von Texten eingebettet ist, aufweist. So kann man in Bezug auf historische Kontroversen und die dabei verwendeten Texte Funktionen wie die Wahrheitsermittlung, den Abgleich von Positionen und von argumentativer Substanz, aber auch die Vertei- 
digung der persönlichen oder der Gruppenehre ansehen. Man muss dabei gar nicht von einer einzigen dominierenden Funktion ausgehen, nicht selten verfolgen die Akteure in historischen Kontroversen mehrere Zielsetzungen gleichzeitig, so dass man dementsprechend von den Kontroversen sagen kann, dass sie zum Beispiel nicht nur der Ermittlung der Wahrheit dienen, sondern gleichzeitig der Verteidigung der wissenschaftlichen Ehre oder von wissenschaftlichem Einfluss.

(2) Sprachliche Handlungen und die möglichen Formen ihrer Sequenzierung, die typisch sind für einzelne Textmuster, sind im Kern von den übergeordneten Funktionen mit bestimmt. So werden wir sehen, dass in den historischen Streitschriften typische Züge wie das Wiedergeben einer gegnerischen Position und die unmittelbar darauf folgende argumentative Behandlung der wiedergegebenen Position z.B. durch Einwände ein häufiges Abfolgemuster ist. Aber auch Formen der persönlichen Herabsetzung gehören in manchen Bereichen zum Repertoire der Handlungsformen.

(3) Ein grundlegender Parameter der Organisation von Kontroversentexten und ganzen Kontroversen ist die thematische Organisation und ihre Dynamik im Verlauf der Kontroverse. So kann man in vielen Kontroversen beobachten, dass eine leitende Streitfrage sich zerlegen lässt in unterschiedliche strittige Teilfragen. Die thematische Substanz einer Kontroverse verändert sich im Lauf der Behandlung der strittigen Punkte. Denn neue thematische Punkte, die etwa zur Stützung einer strittigen Teilfrage eingeführt werden, können ihrerseits zur strittigen Substanz der Kontroverse hinzukommen. Auch die Frage nach den Verfahren und Mustern der Themenorganisation ist ein zentraler Aspekt bei der Analyse von Kontroversentexten und ganzen Kontroversen. So war zum Beispiel das Punkt-für-Punkt-Verfahren, das im Disputationswesen, aber auch im juristischen und im Verwaltungsschrifttum eine lange Geschichte hat, ein wesentliches Verfahren für die Strukturierung der thematischen Substanz und auch der Erfolgsbuchführung in Kontroversen.

(4) Grundlegende kommunikative Maximen wurden zunächst von Paul Grice im Rahmen seiner Theorie der Verständigung und der Implikaturen „entdeckt“. Im Anschluss daran wurde deutlich, dass auch viele weitere Arten von Maximen das sprachliche Handeln in unterschiedlichen Kommunikationsbereichen steuern. Es wurde weiter deutlich, dass die in einzelnen Bereichen gültigen Kommunikationsmaximen auch historisch ausgeprägt und historisch veränderlich sind (vgl. Fritz 2008). So werden wir sehen, dass in unseren Kontroversen immer wieder Prinzipien wie (christliche) Sanftmut, respektvoller Umgang, Höflichkeit und Sachorientierung angemahnt und oft genug auch verletzt wurden. Die Befolgung oder Nicht-Befolgung bestimmter Kommunikationsmaximen ist neben dem Repertoire sprachlicher Handlungen und dem Vo- 
kabular auch ein wichtiger Aspekt der Analyse von persönlichen Kontroversenstilen. Paracelsus oder Jan Abraham à Gehema geben Beispiele für besonders „ruppige“ Kontroversenführung, die sich häufig auch bei Autoren theologischreligionspolitischer Streitschriften findet. Die Berufung auf und die Auseinandersetzung um kommunikative Maximen der Kontroversenführung erlaubt es darüber hinaus, implizite „Kontroversentheorien“ der Zeitgenossen zu rekonstruieren (vgl. Gloning 2005). Solche Theorien der Kontroverse sind im Kern Auffassungen, die zu einer bestimmten Zeit über die Funktionen, die Handlungsspielräume, die Beteiligungsrollen und die Leitprinzipien der Kontroversenführung geherrscht haben. Sie sind historisch veränderlich, ihre Geltungsbereiche können in verschiedenen kommunikativen Domänen auch unterschiedlich ausgeprägt sein.

(5) Historische Textmuster und Gesprächsformen sind auch geprägt von typischen sprachlichen Mitteln, mit denen die zentralen kommunikativen Funktionen umgesetzt werden. Hierzu gehören zum einen typische syntaktische Muster für sprachliche Handlungen (z. B. Vorwürfe) bzw. kommunikative Aufgaben (z. B. Strukturierung einer Aufzählung), zum anderen die lexikalischen Mittel, z. B. für kommunikative Aufgaben im Rahmen der Argumentation wie Konnektoren, aber auch für spezifische thematische Aufgaben. Das Wortschatzprofil von Kontroversen und Kontroversenbeiträgen ist also funktional und thematisch geprägt, es hängt aber auch mit der Befolgung von Kommunikationsmaximen zusammen. Höfliche und respektvolle Rede erfordert in der Regel auch bestimmte lexikalische Mittel, umgekehrt sind Formen der Beleidigung oder der persönlichen Herabsetzung ebenfalls an den Gebrauch bestimmter Ausdrücke gebunden. Die sprachlichen Mittel zeigen besonders deutlich, dass die hier aufgeführten Parameter der Organisation von Texten und komplexen Kommunikationsformen nicht unabhängig voneinander sind, sondern vielfältige Zusammenhänge aufweisen (vgl. Fritz 2016a; 2016b).

(6) Neben den gerade erläuterten Parametern lassen sich noch weitere Gesichtspunkte nennen, die für die Organisation von Kontroversen und Kontroversenbeiträgen erheblich sind, z. B. die Personen- und Rollenkonstellation, Formen der Intertextualität, die Frage nach Phasen der Interaktion, übergeordneten Strategien oder emergenten Resultaten wie z. B. die Bildung von Koalitionen, die ursprünglich gar nicht intendiert waren. Diese Aspekte der Organisation lassen sich auf die ersten fünf grundlegenden Parameter zurückführen. So kann man z. B. thematische Phasen oder Phasen mit einem spezifischen Handlungsprofil rekonstruieren.

(7) In einer historisch-evolutionären Perspektive können wir zunächst nach den Verhältnissen zu einem historischen Zeitpunkt bzw. in einem historischen Zeitschnitt, z. B. der Zeit um 1600, fragen. Dabei sind unterschiedliche 
Stufen der Komplexität zu berücksichtigen: Man kann nach der Organisation eines einzelnen Kontroversenbeitrags, zum Beispiel einer Streitschrift, fragen, man kann darüber hinaus fragen, ob es zu diesem Zeitpunkt so etwas wie ein typisches Muster für die Gestaltung und die Organisation von Streitschriften gibt. Auch die Frage, ob und ggf. welche Gemeinsamkeiten oder Unterschiede solche Muster in den unterschiedlichen Fachzonen aufweisen, ist wichtig für die Analyse der Verhältnisse zu einem bestimmten Zeitpunkt. Dieselben Frageperspektiven kann man auch auf die nächsthöhere Konfigurationsebene anwenden, die Kontroverse als einen Verbund von Kontroversenbeiträgen, der zum einen durch einen thematischen Fokus, zum anderen durch intertextuelle Verweistechniken strukturiert ist. Und auch hier kann man über den Vergleich unterschiedlicher Kontroversen herausfinden, ob und inwiefern es ein für den Zeitschnitt typisches Organisationsmuster wissenschaftlicher Kontroversen gibt oder ob es solche Organisationsmuster nur für bestimmte Fachzonen gibt. Für die Zeit um 1700 kann man zum Beispiel zeigen, dass die Nutzung von Zeitschriftenartikeln die Voraussetzung war für ein verändertes Kontroversenmodell, dass aber das ältere Streitschriftenmodell noch lange Zeit parallel zum neuen Modell angewendet wurde.

Damit sind wir auch bei der Frage nach der Entwicklung von Arten von Kontroversenbeiträgen (Texttypen, Gesprächsformen) und Arten der Organisation von wissenschaftlichen Kontroversen im historischen Längsschnitt. Das Bild von Jost Trier zu den Zeitschnitten und ihrer Abfolge bei der Organisation von Wortfeldern lässt sich übertragen auf die Beschreibung der kommunikativen Verhältnisse und ihrer historischen Dynamik. Eine solche Längsschnittperspektive setzt natürlich viele Einzelbefunde zu zeitlich gestaffelten Querschnitten und zu unterschiedlichen Fachzonen voraus: sowohl zur typischen Organisation von Kontroversenbeiträgen als auch von ganzen Kontroversen. Diese Voraussetzungen sind derzeit zwar noch nicht breit erfüllt, aber es liegen doch viele detaillierte Vorarbeiten vor, die ein Bild zu den Verhältnissen im 16., 17. und 18. Jahrhundert ermöglichen und auch erste Entwicklungstendenzen zeigen.

In einer historisch-evolutionären Perspektive stellt sich schließlich auch die Frage nach den Formen und Formaten der Erklärung von historischen Entwicklungen solcher kommunikativen Konfigurationen auf den genannten Komplexitätsstufen. Ich habe oben bereits angedeutet, dass eine Invisible-hand-Theorie den Rahmen für eine Erklärungsumgebung liefern kann, die allerdings angereichert werden müsste mit Komponenten, welche die medialen und institutionellen Rahmenbedingungen stärker mit berücksichtigen.

In den folgenden Abschnitten bespreche ich nun in gebotener Kürze vier unterschiedliche Kontroversen, die unterschiedlichen Zeitschnitten und ver- 
schiedenen Fachzonen angehören. Sie stammen aus dem Umfeld von Forschungen, die ich in den letzten Jahren zusammen mit Gerd Fritz unternommen habe und die im Verlag John Benjamins erscheinen. Nach den vier Beispielen folgen eine erste Zusammenschau und ein Ausblick.

\section{Johannes Kepler in einer Kontroverse über den Status der Astrologie als Wissenschaft (1597-1610/11)}

\subsection{Die Kontroverse: Gegenstand, Beiträger, Texte}

In den Jahren 1609 und 1610 veröffentlichte Johannes Kepler, vielleicht der hochkarätigste Mathematiker und Astronom der Zeit um 1600, zwei Kontroversenbeiträge in deutscher Sprache, in denen es in erster Linie um den Status der Astrologie als Wissenschaft ging. ${ }^{2}$ Dabei bezog er sich zum einen auf Schriften von Helisäus Röslin, einem Hofmann, den er persönlich kannte und der 1597 und 1606 Abhandlungen über Kometen und neue Sterne und ihren Vorzeichencharakter veröffentlicht hatte. In einer lateinischen Schrift aus dem Jahr 1606 hatte sich Kepler kritisch dazu geäußert, was Röslin zu einer Erwiderung veranlasste, die 1609 als selbstständige Schrift erschien. In seinen Werken von 1609 und 1610 antwortet Kepler zum einen auf Röslin, er bezieht sich aber auch auf einen weiteren Kontroversenstrang zu diesem Thema, einer Auseinandersetzung zwischen dem Prediger Melchior Schaerer, selbst Verfasser von Kalendern mit Vorhersagen, und dem Arzt Philipp Feselius, einem scharfen Kritiker astrologischer Vorhersagen. Man kann die Positionen der Befürworter (Röslin, Schaerer) und der Gegner (Feselius; Kepler nur in Teilen) der Astrologie verkürzt und schematisch so wiedergeben.

Die Befürworter der Astrologie und des Zeichencharakters neuer Sterne vertreten folgende Auffassungen:

a) Kometen und Neue Sterne haben Vorzeichencharakter.

b) Spezifische Prophezeiungen sind möglich und methodisch gesichert.

Dagegen sind die Gegner der Astrologie und des Zeichencharakters neuer Sterne der Auffassung:

2 Vgl. zu dieser Kontroverse Gloning 2002 und Fritz/Gloning (to appear), Ch. 2. 
a) Kometen und Neue Sterne haben keinen Vorzeichencharakter.

b) Die Astrologie ist nicht zuverlässig, sie hat keine sicheren Fundamente, ihre Prophezeiungstechnik ist methodisch ungesichert.

Kepler vertritt mit seinen beiden Schriften eine vermittelnde Position, die man wiederum verkürzt mit folgenden Auffassungen charakterisieren kann:

a) Die Himmelskörper beeinflussen die „Niedere Welt“.

b) In der Regel sind keine spezifischen Prophezeiungen ableitbar.

c) Das „Forschungsprogramm Astrologie“ als Frage nach kosmologischen Zusammenhängen verdient weitergeführt zu werden, auch wenn noch keine sicheren Ergebnisse vorliegen (Frühstadiumsargument) und trotz herrschender Missbräuche.

Folgende Schriften lassen sich dieser Kontroverse zuordnen, die eingerückten gehören zum Schaerer/Feselius-Strang, Keplers „Tertius interveniens“ (1610) verbindet die beiden Kontroversenstränge:

1597 Röslin, Tractatus

1605 Röslin, Judicium

1606 Kepler, De stella nova in pede serpentarii (I + II)

1607/8 \& 1608/9 Schaerer, Vorreden zu Prognostica (mit Bezug auf mündliche Diskussionen)

1609 Röslin, Historischer Diskurs von heutiger Zeit Beschaffenheit

1609 Kepler, Antwort auf Röslini Diskurs

1609 Feselius, Gründlicher Diskurs von der Astrologia Judiciaria 1610 Kepler, Tertius Interveniens

1611 Schaerer, Verantwortung und Rettung

1612 Röslin, Mitternächtige Schiffahrt, Cap. 12

Die doppelsträngige Kontroversenstruktur ist in der folgenden Abbildung veranschaulicht, wobei die Pfeile jeweils „reagiert auf“ bedeuten. 


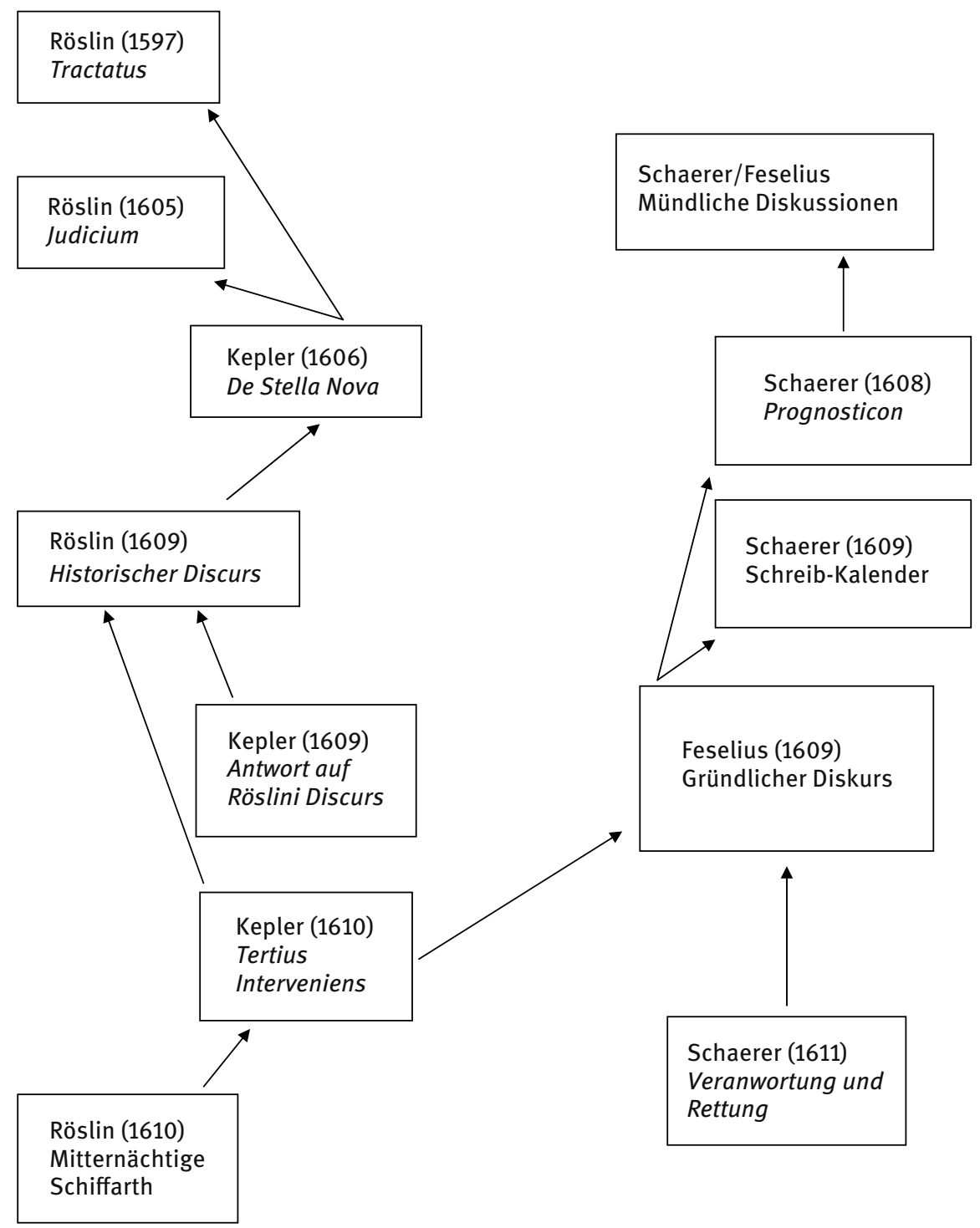

Abb. 1: Die Beiträge zur Kontroverse über den Status der Astrologie zwischen Röslin, Kepler, Schaerer und Feselius (eigene Abbildung). 


\subsection{Personenkonstellation und Ziele}

Betrachtet man nun zunächst die Personenkonstellation und die Ziele der Beteiligten, dann erkennen wir drei „Lager“, die Befürworter und die Gegner der Astronomie sowie Kepler als Vertreter einer vermittelnden Position. Im Hinblick auf die Ziele der Opponenten muss man als vierte Gruppe auch noch die Leserschaft, darunter auch Landesherren und einflussreiche oder persönlich wichtige Personen, berücksichtigen. Die Ziele der Beteiligten lassen sich grob in drei Dimensionen erkennen:

(i) das Streben nach Wahrheit und Erkenntnis, dabei wird der Gegner in der Kontroverse als Partner im Erkenntnisstreben angesehen;

(ii) die Verteidigung der eigenen, persönlichen Ehre;

(iii) die Verteidigung und Propagierung der eigenen Auffassung; dabei wird der Gegner als unüberzeugbar angesehen, Ziel ist es nicht, den Gegner zu überzeugen, sondern Dritten gegenüber den eigenen Standpunkt zu vertreten.

\subsection{Streitschriften und das Punkt-für-Punkt-Verfahren}

Die zentrale Darstellungsform in der Kontroverse ist die Streitschrift. Allerdings wurde der Anlass der Kontroverse, das Strittige, in Texten anderer Art hervorgebracht, zum einen in Passagen, die in Abhandlungen eingelagert waren (Röslin, Kepler), zum anderen mit kritischen Textteilen in prognostischen Schreibkalendern, mit denen Schaerer auf mündliche Unterredungen Bezug nimmt. Die Streitschriften, die Kepler nutzt, folgen im Hinblick auf den Textaufbau, die Textbausteine und die thematische Organisation einem etablierten Schema. Ein wesentliches Kennzeichen ist das thematische Prinzip der Punktfür-Punkt-Zerlegung, wie wir es auch in religionspolitischen Kontroversen der Zeit finden (Gloning 1999). Dabei wird der Streitgegenstand in unterschiedliche Teilpunkte zerlegt, die jeweils für sich und der Reihe nach bearbeitet werden. Dabei werden spezifische Verfahren zur Kennzeichnung der Textgliederung und der Markierung von Textteilen genutzt, z.B. Wendungen wie Das ander Argument im Sinne von, der zweite Streitpunkt‘.

Auch für die Behandlung einzelner Punkte gibt es ein etabliertes Verfahren, das darin besteht, die Auffassung des Gegners zunächst wörtlich oder inhaltlich wiederzugeben, um dann darauf zu reagieren. Das folgende Textbeispiel für dieses Wiedergabe-und-Antwort-Format stammt aus Keplers Schrift „Tertius Interveniens“. In der linken Randspalte habe ich den Wiedergabe-Teil Keplers - Kepler gibt wieder, was Röslin über Kepler sagt - mit „,W]“ markiert, den Antwort-Teil Keplers mit „[A]“, diese Markierungen stammen von mir. Im 
Text selbst kennzeichnen die Ausdrücke „Ibid.“ den Verweis auf eine Textstelle bei Röslin und „Ant.“ die Entgegnung Keplers. Die Streitschriften bestehen im Kern aus einer Sequenz solcher Wiedergabe-Antwort-Bausteine.

[W] Ibid. [= Fol. C iij a] Kepler sagt/ das die Erd vmblauffe/ das sey nit wider die schrifft/ wann man sie recht außlege: diese auslegung wollte D. Röslin gern vernemen.

[A] Ant[wort]. So höre er darüber alle Bäpste von $1542 \mathrm{an} /$ die haben die schrifft also ausgelegt/ das sie Copernicum, vnangesehen derselbig sein Opus Revolutionum Paulo III. dedicirt/ noch nie eins Irthumbs oder Ketzerey beschuldiget: Er höre darüber etliche fürneme Theologos deren anderen Partheyen/ so man Protestantes nennet/ die nemen jhn kein gewissen diß zu glauben/ vnd ist doch zuvermuthen/ sie machen jhn so enge gewissen vber die meynung Heiliger schrifft/ als D. Röslin nimmermehr. Er höre auch die Mathematicos als laicos, wie sie sich wegen der Heiligen schrifft entschuldigen: sonderlich Maestlinum, wellichem D. Röslin billich hoch helt. Ob wol nicht ohn das hingegen der grosse hauff von Theologis vnd Mathematicis auß allen Partheyen es mit Röslino halten/ vnd gar nit zu geben wöllen das die Erd vmblauffe. Entlich so besehe D. Röslin die prolegomena meiner Astronomiae novae seu Commentarij de Marte, so er gelegenheit hat/ da wirdt er etwas finden/ von dem jenigen/ das er so gerne vernäme. (KGW 4:106.16 ff.)

\subsection{Das Repertoire sprachlicher Handlungen}

Man kann an diesem Textbeispiel auch sehen, wie in Streitschriften einzelne thematische Punkte kombiniert werden mit spezifischen Arten von sprachlichen Handlungen. In diesem Punkt steht die Teilfrage zur Diskussion, ob die Auffassung, dass die Erde nicht stillsteht, mit der Bibel verträglich ist. Kepler antwortet darauf mit einer Reihe von Autoritäten, von denen er annehmen kann, dass sie auch für seinen Gegner akzeptabel sind.

Das Repertoire der sprachlichen Handlungen und der Handlungssequenzen in Streitschriften weist eine beträchtliche Vielfalt und Komplexität auf. In einem ersten Schritt kann man die Handlungsweisen in vier größeren funktionalen Gruppen zusammenstellen:

(i) eine (eigene oder gegnerische) Position formulieren;

(ii) etwas (eine Position, einen Teilpunkt, ein angreifbares Argument) stützen;

(iii) eine gegnerische Position, einen gegnerischen „Punkt“ angreifen;

(iv) die Person des Gegners angreifen oder herabsetzen.

Die einzelnen Gruppen sind intern reich ausgebaut, vielfach differenziert und weisen eine Fülle von Realisierungsformen und Sequenzierungsmöglichkeiten auf. Sie können an dieser Stelle nicht in Breite behandelt werden. Eine ausführ- 
lichere Darstellung des Repertoires sprachlicher Handlungen in dieser Kontroverse bietet das zweite Kapitel in Fritz \& Gloning (to appear). Ein einzelnes Beispiel soll immerhin zeigen, wie ein komplexer Argumentationszusammenhang durch Indikatoren auf der Textoberfläche nachvollziehbar gemacht wird.

Im folgenden Textbeispiel aus Keplers „Tertius Interveniens“ (1610) wird eine zunächst formulierte These mit einer strukturierten Reihe von fünf ProArgumenten gestützt. Die Struktur des Abschnitts wird mit sprachlichen Indikatoren auch an der textuellen Oberfläche gekennzeichnet: Mit „Daß aber ...“ wird die These eingeleitet, mit „Dann“ wird signalisiert, dass nun eine Stützung folgt, die intern mit „erstlich“, „Zum andern vnd ferrners“ usw. weiter unterteilt ist:

LXIV.

(...)

Daß aber auch der Mensch mit seiner Seel vnnd deroselben nideren Kräfften ein solche Verwandtnuß mit dem Himmel habe wie der Erdtboden/ mag in viel wege probiert vnnd erwiesen werden: deren ein jedweder ein Edels Perl auß der Astrologia ist/ keines wegs mit der Astrologia zu verwerffen/ sondern fleissig auffzubehalten vnd zu erklären.

LXV.

Dann erstlich mag ich mich dieser Experientz mit Warheit rühmen/ daß der Mensch in der ersten Entzündung seines Lebens/ wann er nun für sich selbst lebt/ vnnd nicht mehr in Mutterleib bleiben kan/ einen Characterem vnd Abbildung empfahe totius constellationis coelestis, seu formae confluxus radiorum in terra, vnd denselben biß in sein Grube hieneyn behalte: (...).

LXVI.

Zum andern vnd fermers/ (...)

LXVII.

Fürs dritte ist diß auch ein wunderlich Ding/ daß die Natur (...).

LXVIII.

Zum vierdten/ (...)

LXIX.

Endtlich vnd zum fünfften (...)

(KGW 4:209 ff.; Fett-Hervorhebung T. G.)

Diese und weitere Verfahren der internen Kennzeichnung der thematischen Organisation und von Aspekten der Handlungsstruktur sind nicht untypisch für Streitschriften. Sie hängen eng zusammen mit dem Punkt-für-Punkt-Verfahren der thematischen Zerlegung und der damit verbundenen Systematisierung in der Behandlung kontroverser Substanz und ihrer Dynamik im Verlauf der Auseinandersetzung. 


\subsection{Kommunikationsmaximen und ein implizites Kontroversenideal}

In den Kontroversenbeiträgen finden sich immer wieder reflexive Passagen, in denen die Autoren Aspekte der Auseinandersetzung selbst zum Gegenstand machen und Prinzipien der Kontroversenführung thematisieren. Solche Passagen lassen sich im Rahmen einer historisch verlängerten Theorie der Kommunikationsmaximen behandeln, sie sind eine wertvolle Quelle für die Rekonstruktion von impliziten Kontroversenidealen (vgl. u. a. Fritz 2008; Gloning 2005). Der Zusammenhang besteht darin, dass sich von „Ordnungsrufen“, die in Texten formuliert werden, auf die zugrundeliegenden Kommunikationsprinzipien, die damit eingeklagt werden, rückschließen lässt. Wenn also ein Autor seinem Gegner vorwirft, dieser habe ihn in einem Kontroversenbeitrag nicht mit dem nötigen Respekt behandelt, dann legt er sich dabei auf eine Maxime der respektvollen Behandlung unter Kontroversengegnern fest. Wir werden gleich sehen, dass solche Ordnungsrufe, die damit verbundenen Maximen und die Ausführungsbestimmungen dazu auch Gegenstand der Aushandlung und der Auseinandersetzung sein können. Der folgende Text ist ein Beispiel dafür. Kepler reagiert damit auf einen Vorwurf Röslins, er, Kepler, habe ihn schumpfiert, also ungebührlich behandelt. Kepler rechtfertigt sich, indem er das Prinzip der Höflichkeit und der schonenden Milderung einem Prinzip der wissenschaftlichen Direktheit und Klarheit gegenüberstellt, das nach seiner Auffassung höherrangig ist:

(...) Das ich aber nit viel Caeremonias Academicas, oder Titulirns gemachet/ sondern ohne scheuh mit worten außgesprochen/ wie Ichs im Hertzen empfunden: bekenn ich gern/ (...) vnd Ich nit allein in der erfahrung befunden/ das die verständige Lesere kein gefallen an sollichen Parergis vnd fuchsschwäntzen [höfliches, schmeichelhaftes Drumherumreden; T.G.] haben: sondern ich es auch an einem Philosopho für einen vbelstand halte/ wann er sein mainung de rebus scientificis (...) mit viel verdräeten worten einigem lebenden zugefallen verduncklen wolte. (KGW 4:116.21 ff.)

Er gibt in einer späteren Passage auch eine funktionale Begründung dafür: Harte und direkte, ungemilderte Ausdrucksweise ist förderlich für den wissenschaftlichen Fortschritt. Er beschreibt in diesem Zusammenhang die kompetitive Diskussionskultur unter den Wissenschaftlern am kaiserlichen Hof in Prag und kontrastiert sie mit den „schonenden“ Umgangsformen in Röslins höfischer Umgebung. Kepler hebt dabei die wesentliche Rolle von harten Diskussionen für seinen eigenen intellektuellen Fortschritt hervor, die unter anderem darin besteht, dass man dadurch ein umstrittenes Kontroversengebiet sehr gut kennenlernt, die strategische Gewichtung von Argumenten gesprächsweise 
erproben und die Resultate in späteren Publikationen nutzen kann. Kepler schreibt:

Jch aber hab allhie zu Prag einen härtern stand/ vnd kom ich zu sollichen promptis vnd vividis ingenijs nidrigern stands/ deren allzeit alhie ein gute anzahl die mir nit vil Cramantzens machen/ sondern fein trocken sagen/ wie sie es meinen/ wort vmb wort geben/ vnd es so lang treiben/ biß einer den andern vberwindet: (...) Wann ich mit sollichen super astrologicis disputire, da hab ich böß machen/ vnd werde also exercirt, das ich sie wol mag meine Lehrmeister nennen. Dann wider die astrologiam haben sie materiam dicendi copiosissimam, wie auch wider allerhand Bedeuttungen. Soll ich etwas wider sie erhalten/ vnd die astrologiam nit gar verlieren/ so muß ich mit verwerffung oder beyseittsetzung dessen/ so etwas vngewiß/ jnen vorkommen/ vnd die Vorstatt verbrennen/ damit ich die Vestung erhalte: hernach aber in offnen schrifften/ dessen so ich verlohren geben/ ingedenck sein/ vnd der philosophiae standhafftigkeit in acht nehmen. (KGW 4:128.7 ff.)

Man sieht an diesem Textbeispiel also, wie sich an den Vorwurf der Unhöflichkeit bzw. der ungebührlichen Behandlung reflexive Passagen anschließen, in denen die Konkurrenz unterschiedlicher Maximen, hier die Höflichkeitsmaxime vs. die Maxime der ungeschützten und direkten wissenschaftlichen Rede, diskutiert wird. Bringt man diese reflexiven Stellen in einen Zusammenhang, dann lässt sich auf dieser Grundlage eine implizite Kontroversentheorie rekonstruieren, die für die einzelnen Beteiligten leitend ist im Hinblick auf ihr Handeln im Rahmen von wissenschaftlichen Auseinandersetzungen. Für Kepler und seine Kollegen gehören zu diesen Maximen:

- das Prinzip der Wahrhaftigkeit und der Redlichkeit;

- das Prinzip des respektvollen, sachbezogenen Umgangs mit dem Gegner (zu dessen Ausführungsbestimmungen aber auch wissenschaftliche Direktheit gehören kann);

- das Prinzip der wohlwollenden Deutung von Äußerungen des Gegners;

- das Prinzip der Klarheit, der Verständlichkeit und der Eindeutigkeit;

- das Prinzip der (zuweilen) witzigen Darstellung;

- das Prinzip der Kürze bzw. der ökonomischen Darstellung.

Das Prinzip der (zuweilen) witzigen Darstellung würde man vielleicht so nicht erwarten im Bereich der Wissenschaft. Aber Kepler formuliert es an einer Stelle im Vorwort zu „Tertius interveniens“ sogar explizit, wenn er sagt, sein Ziel sei es, „nur allein die Warheit zu ergründen/ vnnd dem Leser die Philosophiam mit etwas Frölichkeit eynzubringen/ darvmb sich andere streittige Haderkatzen nichts anzunemmen" (KGW 4:149.20). Und es gibt auch witzige Beispiele in seinem Text selbst. So reißt er einmal einen Röslin-Satz aus seinem Zusammenhang, in dem es um die Theorie der Entstehung von Regen geht. Aus dem 
Zusammenhang gerissen lautet der Röslin-Satz sehr trivial („Der Regen kommt von oben“), Kepler kann ihn seinem Gegner leicht „zugestehen“ und auf witzige Weise erweitern:

D. Röslin. Die gute fruchtbaren regen kommen von oben herab. Keppler. Jst war/ sonst wurden die Kühe an Beuchen naß/ wann es vbersich regnete. (KGW 4:111.20 f.) ${ }^{3}$

Damit verlassen wir diese Kontroverse. Man kann wohl sagen, dass Kepler sein vermittelndes Ziel erreicht hat, zum einen die Kritik an den genauen astrologischen Vorhersagen als berechtigt zu erweisen, zum anderen aber das (kosmologische) Forschungsprogramm, bei dem es ihm um die Frage nach dem Zusammenhang der „oberen“ und der „unteren Welt“ geht, zu retten.

\section{Eine Kontroverse über medizinische Reformen um 1700: Behandlungsmethoden und Berufsethos der Ärzte}

Im Jahr 1688 veröffentlichte Jan Abraham à Gehema eine harsche Kritik an traditionellen medizinischen Behandlungsmethoden in einer deutschsprachigen Streitschrift. ${ }^{4}$ Dabei kritisiert er nebenbei in scharfer Weise auch das Berufsethos der Ärzte, vor allem - in Gehemas Perspektive - deren Geldgier, Verantwortungslosigkeit und mangelnde fachliche Einsicht. Schon auf dem Titelblatt werden die zentralen Gegenstände der Kritik benannt, der Aderlass, das Schröpfen, das Purgieren und Klistieren sowie der Gebrauch von Kühltränken und herzstärkenden Mitteln, die allesamt als „Grausame Medicinische Mord-Mittel“ bezeichnet werden. Ein Jahr später, im Jahr 1689, erschien eine Verteidigungsschrift des Arztes Melchior Geuder, die ebenfalls in deutscher Sprache abgefasst war. Man erkennt bereits an der quasi-symmetrischen Anlage des Titelblatts und am kontrastierend gebrauchten „Heilsame Medicinische Lebens-Mittel“, dass hier ein argumentatives Gegenprogramm entworfen wird.

3 ,Das ist wahr, sonst würden ja die Kühe an den Bäuchen nass, wenn es in die andere Richtung, von unten nach oben, regnen würde‘.

4 Vgl. zu dieser Kontroverse Gloning/Lüsing 2002 und Fritz/Gloning (to appear), Ch. 4. 


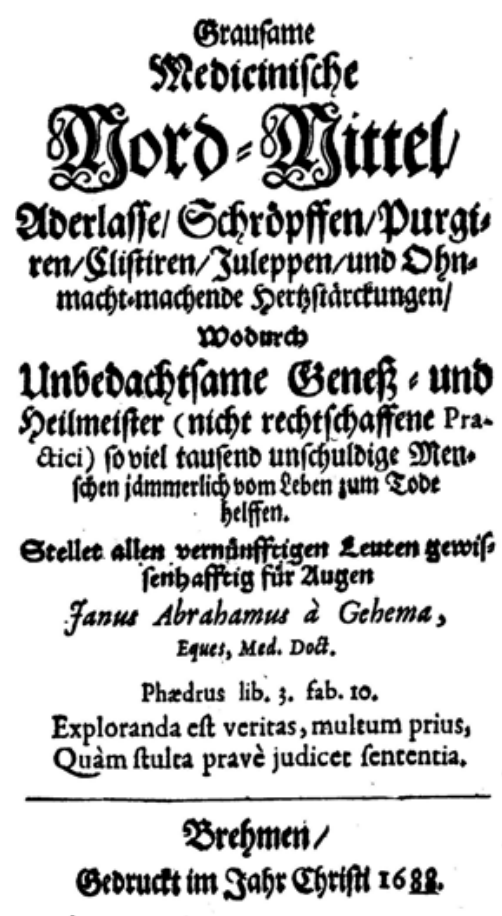

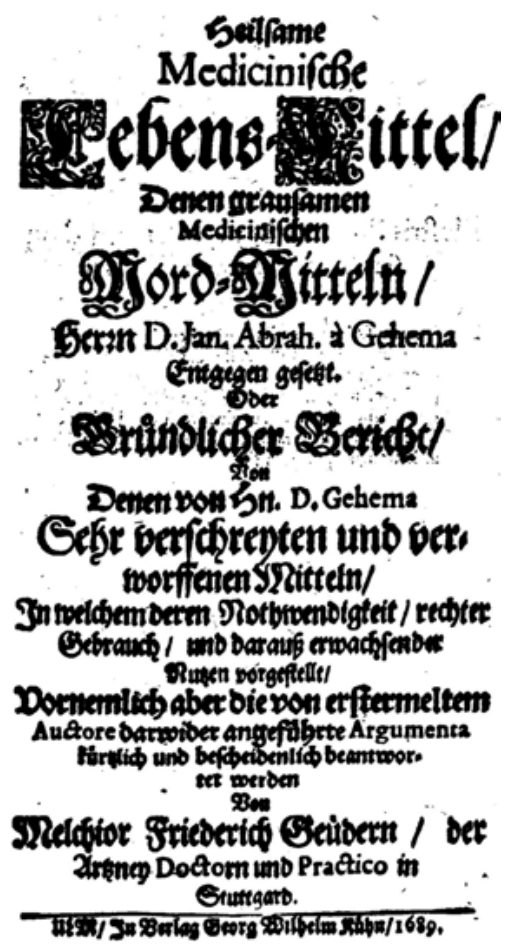

Abb. 2: Titelblätter der Streitschriften von Gehema (1688) und Geuder (1689).

Quelle: Faksimile Antiqua-Verlag 1980.

\subsection{Thematische Zerlegung in Streitpunkte und Verfahren der Organisation textueller Bezüge}

Im Kern ist Gehemas Streitschrift eine Abfolge von mehreren unterschiedlich langen Abschnitten, die jeweils einer der erwähnten traditionellen Behandlungsmethoden gewidmet sind. In diesen Abschnitten werden u. a. die traditionellen Auffassungen wiedergegeben und mit ganz unterschiedlichen Formen der Kritik begleitet, z. B. mit Einwänden oder Rückfragen unterschiedlicher Art. Das folgende Textbeispiel findet sich im Abschnitt mit Gehemas Kritik am Purgieren, der Nutzung starker Abführmittel, die nach traditioneller Auffassung der Reinigung des Körpers dienen sollte. Ich habe die Wiedergabe der traditionellen Auffassung mit „[T]“ (These) gekennzeichnet, Gehemas kritische Einwände bzw. Rückfragen dann mit „[E]“ und einer Ziffer. Wir werden später sehen, dass Geuder in seiner Antwort diese innere Struktur abarbeitet.

Als dritte und nechst der Aderlasse grausame Medicinische Mord-Mittel ist das außmergelende Purgieren: und nach dem Rang das zweyte / wo nicht das erste Helff-Mittel / wel- 
ches unsere Herrn Titul-Doctores, so bald dieselbe zu einem Patienten vociret werden / praesentiren / [T] welches sie insonderheit darumb ordiniren / weil sie mit der falschen Einbildung praeoccupiret sind / als ob der Leib dermassen unflätig und unrein sey / daß er müsse gereiniget werden? Ich frage aber diese vorsichtige und curieuse Herrn / [E1] was sie für indicia sothaner Unreinigkeit haben / [E2] und auff was Art und Weise sie den Leib reinigen können / ohne die darinn vorhandene andere nützliche Säffte zugleich außzuführen. (Gehema $36 \mathrm{f}$.)

Das Textbeispiel zeigt auch, wie Gehema bereits bei der Wiedergabe der gegnerischen Auffassung mit kritischem (ausmergelnd) und zum Teil auch herabsetzendem (,unsere Herrn Titul-Doctores“) Vokabular nicht spart. Diese polemischen Mittel, auf die ich unten noch genauer eingehe, gehören mit zum persönlichen Kontroversenstil Gehemas. In seiner Erwiderungsschrift greift Geuder die beiden Einwände auf und beantwortet sie in strukturierter Weise. Auch diese Antwort besteht zunächst in einer Wiedergabe, die eine Textreferenz (,am 48. Blatt“) und dann eine Paraphrase der von Gehema vorgelegten kritischen Rückfragen enthält (im Zitat von mir markiert mit „[W1]“ und „[W2]“). Er verbindet diese Wiedergabe seinerseits mit der Kritik, dass Gehema diese Punkte nicht näher ausgeführt habe, und beantwortet dann jede der beiden Teilfragen in strukturierter Weise.

So wäre auch zu wünschen / daß er seine am 48. Blatt vorgelegte Frag / [W1] was man nemlich vor Anzeigen habe / daß der Leib unrein / und [W2] auf was Art und Weise man den Leib reinigen könne / ohne die darinnen vorhandene andere nutzliche Säffte zugleich außzuführen? Etwas weitläuffiger vorgestellet / oder gar erörtert hätte. Und sintemahlen dieses eine zwyfache Frage ist / so weise ich / [A1] den ersten Puncten betreffend / den jenigen Leser / welcher dessen Erörterung verlangt / zu der Institutionisten und anderer Auctorum [...] Schrifften und Bücher [...]

[A2] Belangend aber das andere / so ist zu wissen / [A2.1] daß erstlichen nimmermehr ein Medicus sich unterstanden [...] [A2.2] Zu dem anderen / daß das jenige / so etwa bey dem Purgieren [...] [A2.3] Und drittens / daß kein einig medicamentum evacuans [...] (Geuder 61f.)

Man sieht an diesem Beispiel, welche Verfahren Geuder anwendet, um einerseits die Bezüge zur gegnerischen Schrift herzustellen, um andererseits seine eigene Entgegnung auf zwei Ebenen intern zu strukturieren und durch geeignete textuelle Indikatoren für den Leser auch durchsichtig zu machen. Der Bezug zu den beiden kritischen Rückfragen Gehemas wird durch die Wendungen „den ersten Puncten betreffend“ und „Belangend aber das andere“ hergestellt (im Zitat von mir mit „,A1]“ und „[A2]“ gekennzeichnet). Innerhalb der Erwiderung auf die zweite Frage finden wir dann wiederum textstrukturierende Indikatoren, mit denen die Aufzählung unterschiedlicher Argumentationselemente organisiert wird („erstlichen“, „Zu dem anderen“, „drittens“). 
Diese Formen der thematischen und funktionalen Zerlegung der Kontroversensubstanz (Positionen, Pro-, Kontra-Argumente usw.) und die sprachlichen Verfahren für die Organisation der textuellen Bezüge zwischen den Streitschriften sind auch um 1700 noch ganz typisch für das Streitschriften-Modell der Kontroverse.

\subsection{Geuders Rechtfertigung der Sprachwahl}

Für seine Kritik an den traditionellen medizinischen Behandlungsmethoden wählte Gehema eine Streitschrift in deutscher Sprache. Er kommentiert diese Entscheidung in seinem Text nicht weiter. Man kann dafür mehrere Beweggründe annehmen. Zum einen hat die Wahl der Volkssprache eine gewisse Tradition in Reformbewegungen wie der religiösen Reformation, im Paracelsismus oder auch im Umfeld van Helmonts, in dessen medizinischer Reformtradition sich auch Gehema verortet. Dies hängt eng mit der Zielgruppe und dem Gegenstand zusammen, denn Gehema schreibt gegen die aus seiner Sicht geldgierigen und unverantwortlichen Ärzte auch zum Schutz der „einfältigen“, also ungebildeten Patienten (Gehema 1688: 67). Schließlich gibt es in einem etwas späteren Werk, dem „Zeit-Vertreib“ (1689), eine Passage, in der Gehema seine Sprachwahl explizit kommentiert: In Städten wie Danzig, seiner Heimatstadt, lebten neben alten Familien und Gelehrten auch viele Kaufleute und Finanzpersonen, um dieser gemischten Bevölkerung die Lektüre $\mathrm{zu}$ ermöglichen, habe er das Werk nicht in lateinischer, sondern in deutscher Sprache geschrieben (1689: A5a). Vergleichbare Motive dürften auch bei seiner Streitschrift von 1688 die Sprachwahl bedingt haben.

Für Geuder stellte eine Veröffentlichung in deutscher Sprache allerdings ein Problem dar, wie er in seinem Text eingangs schreibt. Die Sprache der Wahl für den gelehrten Arzt ist um 1700 nach wie vor das Lateinische. Eine deutschsprachige Schrift wie die von Gehema würde er unter anderen Umständen gar nicht beachten. Geuder rechtfertigt in diesem Zusammenhang seine eigene Wahl der Volkssprache mit der Tatsache, dass Gehema mit seiner Schrift bereits einen weiten Leserkreis habe, den man für eine notwendige Erwiderung nur erreichen könne, wenn man ebenfalls in deutscher Sprache schreibt. Ich zitiere diese Passage in einiger Länge, weil sie die sprachliche Situation und das Verhältnis von Deutsch und Latein in diesen Jahren sehr gut darstellt.

Es wird sich vielleicht einer und der andere verwundern/ daß ich mir die Zeit vnd Müh nehme/ obenberührtes Herrn Doct. Gehemae Tractätlein zu beantworten (...) Sonderlich wird es einige meiner guten Freunde befremden/ welche wissen/ daß ich vielen in Teutscher Sprach geschriebenen Artzney-Büchern nicht allzugewogen/ und daß ich mir 


\begin{abstract}
vorgenommen/ nimmermehr etwas/ so in Teutscher Sprach geschrieben/ in den Truck zu geben. Dieses ist gewiß/ daß/ wo Herr D. Gehemae Tractätlein in Lateinischer Sprach geschrieben wäre/ sich niemand die Müh hätte nehmen därffen es zu widerlegen/ weilen es vermuthlich von niemand/ als der Sachen verständigen gelesen wäre worden/ welche sich an solche Schmälereyen wenig würden gekehret haben: Sintemalen es aber in unserer Muttersprach geschrieben/ also vielen die der Sachen nicht sattsamen/ ja theils gar keinen Grund und Bericht haben/ über das auch die Sach so cruel und odios vorgestellt/ daß mancher wol von rechtschaffenen Medicis, die sich der von ihme verworffenen Mittel/ etwa bey einem Patienten/ der auß seinen sondern Ursachen/ nicht aber eben/ weil dieses oder jenes gebraucht worden/ es mit der Haut bezahlen muß/ bedienen/ widrige Gedancken schöpffen möchte/ zumalen ich schon in acht genommen/ daß das Tractätlein starck abgegangen/ und von zerschiedenen mit zimlichem applausu aufgenommen worden: Als habe vor nothwendig erachtet/ daß die Sach untersucht/ gleichfalls in unserer Edlen Muttersprach recht und gründlich vor Augen gestellet/ und also einem und dem andern erwachsene zweifelhafftige und widrige Gedancken benommen/ und die Ehr unserer Edlen Medicin, und so vieler dapfferer Medicorum in etwas gerettet werden möchte. (Geuder B1b f.)
\end{abstract}

Man erkennt an der Formulierung ,in unserer Edlen Muttersprach“, dass der Bevorzugung des Lateinischen für fachlich-medizinische Zwecke eine rein funktionale Differenzierung der Sprachwahl zugrundeliegt, die mit einer Hochund Wertschätzung des Deutschen für andere kommunikative Bereiche durchaus gut verträglich ist.

\title{
4.3 Aus dem Repertoire der Handlungsformen
}

Die Gehema/Geuder-Kontroverse ist reich an ganz unterschiedlichen Handlungsformen und Formen der Sequenzierung von sprachlichen Handlungen. ${ }^{5}$ Ein Kernbereich besteht darin, medizinische Praktiken zu kritisieren (Gehema) und medizinische Praktiken zu verteidigen (Geuder). Hier finden wir ein breites Spektrum an argumentativen Verfahren. Auf der Seite von Gehema finden sich auch mehrfach Züge, mit denen der Autor den Besitz der Wahrheit für sich reklamiert und seine Einsicht auf besondere göttliche Gnade und Erleuchtungen zurückführt. Gehema nutzt auch viele Formen der persönlichen Herabsetzung seiner Gegner aus dem Lager der traditionellen Medizin. In dieser Reformkontroverse spielen weiterhin Gewährsleute und Autoritäten eine zentrale Rolle. Deshalb finden sich viele Züge, mit denen traditionelle Autoritäten in der Tradition der Vier-Säfte-Lehre angegriffen oder verteidigt werden, mit denen neuere Gewährsleute eingeführt und als Autoritäten legitimiert werden

5 Für eine ausführlichere Darstellung des Repertoires der Handlungsformen in dieser Kontroverse vgl. Fritz \& Gloning (to appear), Kap. 4. 
(z. B. van Helmont), mit denen aber auch über den Stellenwert von Autoren verhandelt wird.

Aber auch rhetorische Strategien spielen eine wichtige Rolle, zum Beispiel Vergleiche als Mittel der Anschaulichkeit oder für Zwecke der Polemik. So nutzt Gehema zum Beispiel einen absurden Vergleich, um die traditionellen medizinischen Praktiken als abwegig zu kennzeichnen:

Mich wundert daß solche Leuthe nicht lassen ihre Hüte grösser machen als ihr Kopff ist/ oder die Füsse nicht lassen umbher beschneiden/ weil ihnen die Schuhe zu enge sind/ ... (Gehema 3)

Aus der Vielzahl der Handlungsmöglichkeiten möchte ich das Verfahren der Fallunterscheidung herausgreifen und zeigen, wie es im Rahmen einer Kombinatorik von Verteidigungszügen eingesetzt wurde. Der folgende Textauszug aus Geuders Schrift umfasst zunächst die Wiedergabe einer gegnerischen Position mit einem Kritikpunkt, sodann die Unterscheidung verschiedener Fälle, die der Verteidigung zugrundeliegt (Unterscheidung milder Gebrauch vs. Missbrauch), sodann eine Gegenbehauptung, die auf der Differenzierung der Fälle beruht, auf der Unterscheidung von mildem Gebrauch und Missbrauch. Mit „[W]“ und „[A]“ kennzeichne ich wiederum den Wiedergabe- und den Antwortteil des Textbausteins:

[W] Was ferners am 54. und folgenden Blättern von Beraubung deß so sehr dienlichen muci intestinorum, von denen nach eingenommenen purgierenden Mitteln sich ereignenden greulichen und scheußlichen symptomatibus und Zufällen/ ja gar einem jähen schnellen darauf erfolgenden Tod gemeldet wird; [A] Gehet diejenige Medicos, welche sich gelinder purgirender Mittel in gebührender dosi und Zeit bedienen/ gantz und gar nicht an: alldieweilen dergleichen Zufälle einig und allein vom unbedachtsamen Mißbrauch/ oder mit unterloffenen groben unverantwortlichen Fehlern herrühren: Uber daß dergleichen traurige und leydige Exempel in geringer Anzahl zu finden/ da man hingegen denen selben/ so viel tausend andere/ da das Purgieren glücklich abgegangen/ kan entgegen setzen. (Geuder 63 f.)

\subsection{Gehemas polemisches Kontroversen-Profil}

Schon bei der ersten Lektüre von Gehemas Schrift fällt auf, dass der Autor einen auch für heutige Maßstäbe rauen und polemischen Kontroversenstil praktiziert. Man kann dieses persönliche Profil, das Geuder mehrfach kritisiert und als unangemessen bezeichnet, vor allem auf folgende Aspekte seiner kommunikativen Praxis zurückführen.

Gehema nutzt bei der Bezugnahme auf die Vertreter traditioneller medizinischer Auffassungen ein reiches Arsenal von herabsetzenden Personenbezeich- 
nungen, die sich auf unterschiedliche Aspekte des medizinischen Berufsstandes beziehen. $\mathrm{Zu}$ diesen gehören unter anderem:

Titul-Doctor, Grillen- und Brillen-Verkäufer, Träumer, Galenischer Suppen-Doctor, die Verkehrten, Idiot, Tyrann, Blutvergiesser, Schwätzer, Humorist (,Anhänger der Säftelehre', ein traditionelles Schimpfwort der Gegner), Mährlein-Krämer und viele andere.

In vergleichbarer Weise nutzt Gehema eine breite Palette von herabsetzenden und kritischen Bezeichnungen für medizinische Handlungsweisen, die Situationen und Resultate ihrer Anwendung, aber auch für die zugrundeliegende Berufsauffassung von Ärzten (z. B. Schlendrian). Hierzu gehören unter anderem:

Mord-Mittel, jämmerlich vom Leben zum Tode helfen, Schlendrian, Fehler, irrationale Methode, Narrenteidung, Ränke, Mißbräuche, martern, peinigen, tyrannisieren, calumnieren, schmähen, lästern, verfolgen, Mord (,hier ist offenbahrer Mord“), ermorden, Folter-Banck, Gift-Suppe, ...

Gehemas Personalstil weist viele weitere Spielarten der Polemik als Elemente seines persönlichen Repertoires auf. Er steht damit in der Tradition anderer „Reformer“ der Frühen Neuzeit, z. B. Luther, Paracelsus oder van Helmont. Er ist damit aber auch ein lohnendes Ziel für seinen Gegner Geuder, der die polemischen Züge Gehemas zum Anlass nimmt, Maximen der Streitkommunikation in reflexiver Weise zu kommentieren. Diesem Aspekt der Kontroverse wenden wir uns nun $\mathrm{zu}$.

\subsection{Die Auseinandersetzung um Maximen der Streitkommunikation}

Wie in der Astrologie-Kontroverse um Johannes Kepler haben die Beteiligten in der vorliegenden Medizin-Kontroverse mehrfach Gelegenheit, die Prinzipien der Kontroversenführung reflexiv zu thematisieren. Melchior Geuder hat dafür besonderen Anlass, weil er zum einen in der Kontroverse mit Gehema die reagierende Partei ist und weil ihm zum anderen Gehema vielfältige Anlässe gibt für „Ordnungsrufe“ und für die Berufung auf kommunikative Maximen der fairen, sachgemäßen, respektvollen usw. Kontroversenführung. Auch in diesem Zusammenhang erinnern wir daran, dass Vorwürfe und Ordnungsrufe, die sich auf die Verletzung von kommunikativen Maximen im Rahmen der Kontroversenführung beziehen, wertvolle Hinweise sind, die eine Rekonstruktion von impliziten Kontroversenidealen bzw. -theorien erlauben. In der Gehema/ Geuder-Kontroverse werden unter anderem folgende Maximen ggf. im Anschluss an Verletzungen thematisiert: 
- das Prinzip der Aufgeschlossenheit gegenüber Kritik;

- das Prinzip der verteilten Beweispflicht, womit zum Teil argumentative Verpflichtungen zurückgewiesen werden;

- das Prinzip des respektvollen Umgangs beim Kritisieren, das zum Teil auf christliche Prinzipien der Sanftmut zurückgeführt wird;

- das Prinzip der Aktualität der Kritik („Kritisiere nichts, was zu einem bestimmten Zeitpunkt überhaupt nicht mehr aktuell ist“");

- das Prinzip der wohlwollenden Deutung („Unterstelle deinem Gegner die sinnvollste und argumentativ stärkste Deutung dessen, was er wörtlich geäußert hat");

- mehrere Prinzipien des korrekten, sachgemäßen Argumentierens.

In den folgenden beiden Textpassagen gebe ich Beispiele dafür, wie das Prinzip des respektvollen Umgangs und das Aktualitätsprinzip von Geuder bereits an einer sehr frühen Stelle in seiner Schrift geltend gemacht werden:

Von den anderen beeden Stücken halte ich vor nöthig / etwas mehrers beyzusetzen. Betreffend dann das andere / so stehet es ja allen und jeden vernünfftigen Menschen / sonderlich denen Christen wol an / daß sie in aller Conversation, in allem Leben und Wandel sich einer sanfftmüthigen Art bedienen: Vornemlich aber wird sichs gebühren / daß die / so vor gelehrt und verständig wollen angesehen seyn / andern mit gutem Exempel vorleuchten / und in allen Stucken / sonderlich aber in refutation und Widerlegung sich diese Art lassen befohlen seyn / und einander glimpfflich tractiren. (Geuder A4a)

Ein Prinzip der Aktualität der Kritik kann man in folgender Textpassage erkennen, die ebenfalls früh im Text formuliert wird:

Er [Gehema] beklagt sich hefftig / daß man mehr auf die auctorität der Alten / als auf die Warheit sehe (...) Hätte Herr D. Gehema zu End deß vorigen/ und Anfang dieses Jahrhunderts gelebt/ und dergleichen Sachen geschrieben/ so hätte wol einiger Nutzen können geschaffet werden [...] Allein heutigs Tags wird man dergleichen sehr wenig finden / die sich so steiff und fest an eine Lehre binden und halten sollten. (Geuder A6a f.)

In dieser Passage wird das Prinzip der Aktualität der Kritik nicht explizit formuliert wie im ersten Beispiel (,wird sichs gebühren ... einander glimpfflich tractiren“), sondern es ist rekonstruierbar aufgrund von Geuders Einspruch gegen Gehemas Vorwürfe. Die Analyse der Auseinandersetzung um kommunikative Maximen der Kontroversenführung ist methodisch in zweierlei Hinsicht aufschlussreich. Sie erlaubt es einerseits, einen Beitrag zu leisten zur Beschreibung von persönlichen Kontroversenstilen, sie ist darüber hinaus aber auch der Königsweg zur Ermittlung von impliziten Kontroversentheorien der Zeitgenossen, zu den historisch varianten Auffassungen also, die zu einer bestimmten Zeit über die Funktionen, die Handlungsspielräume, die Beteiligungsrollen 
und die Leitprinzipien der Kontroversenführung geherrscht haben (vgl. hierzu auch Gloning 2005).

\section{Leibnizens Streit mit dem Bergamt um Windenergie und technische Innovationen: Wissenschaft, Technik, Verwaltung (1679 ff.)}

\subsection{Zur Orientierung}

Gottfried Wilhelm Leibniz entwickelte in Hannover am Hof von Herzog Johann Friedrich eine breit angelegte und fruchtbare Tätigkeit, die sich neben Philosophie und Mathematik u. a. auf so vielfältige Bereiche wie Geschichtsschreibung, Rechtswesen, Archiv- und Bibliothekswesen, aber auch auf Fragen der Organisation des Gemeinwesens bezog. Er verfasste in diesem Zusammenhang mehrere umfangreiche Gutachten mit Reform- und Verbesserungsvorschlägen, darunter auch technische Vorschläge zum Bergbau im Harz. Der Bergbau war von erheblicher wirtschaftlicher Bedeutung und mit sog. „Gewerken“ als Form der finanziellen Beteiligung an Investition und Ertrag organisiert. Die Leitung oblag einem Bergamt, das auch die lokale Tradition des Montanwesens verkörperte. Im Bergbau waren in technischer Hinsicht eine Reihe von schwierigen Hauptproblemen vor dem Hintergrund der örtlichen Gegebenheiten zu lösen, dazu gehörten der Umgang mit dem Grubenwasser und die Beschaffung von Förderenergie an den Stellen, wo sie gebraucht wurde, zum Teil tief unter Tage.

Leibniz beteiligte sich in den Jahren 1679 und danach mit Ideen zu technischen Verbesserungen vor allem in zwei Bereichen. Zum einen machte er weitreichende Vorschläge zur Nutzung von Windenergie im Harzer Bergbau. Zum anderen entwickelte er in den Jahren 1692 und danach das Projekt „Seil ohne Ende“, bei dem es in erster Linie darum ging, mit Hilfe einer umlaufenden Seilkonstruktion das enorme Gewicht von sehr langen Eisenseilen zu neutralisieren, das bewegt werden muss, wenn man Stoffe mit einem Einzelseil aus großer Tiefe an die Oberfläche bringen will. Leibniz erfuhr in beiden Fällen heftige Widerstände vor allem von Seiten des Bergamts und seiner Vertreter, die $\mathrm{zu}$ heftigen internen Kontroversen führten.

Diese Kontroversen sind für uns deshalb besonders interessant, weil sie eine enge Verquickung von technisch-wissenschaftlichen Aspekten mit ökonomischen Fragen aufweisen, eine Verbindung, die auch in vielen aktuellen Diskursen eine Grundkonstellation darstellt. Sie sind darüber hinaus wertvoll, 
weil auch diese internen Auseinandersetzungen wesentliche strukturelle Übereinstimmungen mit den textuellen und kommunikativen Verfahren in gedruckten, öffentlichen Kontroversen aufweisen, zum Beispiel das Punkt-für-PunktPrinzip und bestimmte Maximen der Kontroversenführung.

\subsection{Spezifische Teilthemen und Streitgegenstände}

Mit den genannten technischen Themen sind einige besondere Arten von Teilthemen und strittigen Teilfragen verbunden. Hierzu gehören zunächst Fragen der grundsätzlichen technischen Praktikabilität und auch der Umsetzbarkeit unter bestimmten zeitlichen oder finanziellen Rahmenbedingungen:

- (Wie) Ist eine neue Technologie praktisch realisierbar?

- Ist eine neue Technologie in einem bestimmten Zeitraum praktisch realisierbar?

- In welchem Bereich ist eine Technologie anwendbar?

- (Wie) Ist eine neue Technologie finanzierbar und in welchem Verhältnis steht ihre Finanzierung zum Ertrag ihrer Anwendung?

- Ist eine neue Technologie wünschenswert? Hat sie ökonomische Vorteile? Für wen? Gibt es auch Risiken? Für wen?

Ein zweiter Bereich, der strittige Teilfragen aufwerfen kann, ist der Bereich des geistigen Eigentums und der Verwertungsrechte an technischen Innovationen und Erfindungen. Darauf bezogene Fragen sind zum Beispiel:

- Wie identifiziert man „eine Erfindung“ (vor dem Hintergrund einer wissenschaftlichen und technischen Tradition)?

- Wem „gehört“ eine Erfindung? Wessen geistiges Eigentum ist eine Erfindung und wer hat das Recht zur wirtschaftlichen Verwertung? Wie hoch ist ggf. das gratiale, die Vergütung für den Erfinder?

- Wie kann eine Grundidee praktisch umgesetzt werden? In welchem Verhältnis stehen die beiden Leistungen (Grundidee; praktische Umsetzung)?

Ich gebe für diese Arten von Streitgegenständen nun Beispiele aus dem Bestand der genannten Kontroversen, die in verschiedenen Bänden der LeibnizAkademieausgabe (AA) abgedruckt sind (AA I-2-4; AA I-Suppl. Harzbergbau); in der Einleitung zum Supplementband über den Harzbergbau wird erwähnt, dass sich viele weitere Dokumente auch noch in den Archiven befinden. Insgesamt erlauben die edierten Dokumente aber einen sehr guten Einblick in die Art der Kontroversenführung über diese technisch-wirtschaftlichen Fragen. ${ }^{6}$

6 Eine größere Studie zu diesem Verbund wissenschaftlich-technisch-ökonomischer Kontroversen ist in Vorbereitung. 
Im folgenden Textauszug weist Leibniz einen Einwand des Bergamts gegen seinen technischen Neuerungsvorschlag zurück, der sich auf zwei Aspekte bezog, die Praktikabilität des Vorschlags und sein Nutzen/Schaden-Verhältnis. In Bezug auf den Praktikabilitätseinwand beruft sich Leibniz bei seiner Erwiderung auf eine Kommunikationsmaxime, derzufolge Praktikabilitätseinwände auch im technischen Detail ausspezifiziert werden müssen:

Dienet also nichts daß man pro autoritate dahin schreibet, dies oder jenes sey impracticabel, vnd allegiret doch keine difficultät in specie dagegen, item daß man wieder beßer urtheil, vorgeben darff, daß die proposition nicht nutzen, sondern gar schaden bringe, vndt führet doch deßen keine rationes an, ist daß nicht umb die sache herumb gehen vnd die Zeit verlieren? (Leibniz, AA I-4, 67.16 ff.; Juli 1684)

Die folgende Passage stammt aus einem Schreiben des Sekretärs Friedrich Wilhelm Leidenfrost, der Leibniz in vielen Punkten unterstützte. In seinem Schreiben berichtet er über vorgebrachte Einwände, die sich in diesem Fall auf die unterschiedliche Anwendbarkeit der Windenergie im Hinblick auf verschiedene Arten von Gruben bezogen. Es handelt sich hier also um den Gesichtspunkt der Bereichsspezifik der Praktikabilität:

Es waren andere so vorgaben, daß die WM. bey etlichen Gruben wol angehen würden, nemlich die nicht sehr tieff, ob aber bey den allertieffesten, daran zweiffelten Sie. (...) Ew. Herrl. werden es wol deüten, daß Jch alles so candide überschreibe, Es geschiehet blos dem Proponenten [= Leibniz, TG] zum besten, damit derselbe sich darnach in etwas reguliren, und die obstacula desto eher heben könne. (Leidenfrost an Leibniz, AA I-2, 170.1 ff.)

Im Hinblick auf Verfahrensfragen spricht Leidenfrost hier eine wichtige Grundidee der zeitgenössischen Kontroversenlehre an, dass Einwände und kritische Aktivitäten dafür gut sein können, eine Auffassung in der Sache zu verbessern und auch argumentativ im Hinblick auf Einwände besser abzusichern, eine Idee, die uns in ähnlicher Form auch schon früher bei Johannes Kepler begegnet ist.

In der folgenden Passage wird deutlich, dass technische Neuerungen auch Fragen der intellektuellen Priorität und des geistigen Eigentums aufwerfen können. In einem nur internen Schreiben außerhalb des offiziellen Schriftwechsels an den Berghauptmann Otto Arthur von Ditfurdt aus dem Jahr 1693 bezieht sich Leibniz auf einen neuen Vorschlag („Proposition“) zur Verbesserung der Erz-Gewinnung von anderer Seite und fragt dabei nach dem Verhältnis des neuen Vorschlags zu seinen eigenen, früher gemachten Vorschlägen. Er fordert für den Fall, dass seine Grundidee („mein Principium“) in dem neuen Vorschlag aufgegriffen würde, die Möglichkeit $\mathrm{zu}$ „intervenieren“, wobei mir nicht ganz klar ist, ob dies eine Art von Beteiligung am neuen Projekt sein sollte oder eine wie auch immer geartete Form von Einspruch. Leibniz schreibt: 


\begin{abstract}
Nachdem ich nehmlichen vernommen, daß wegen erleichterung des Ertz-treibens eine Neue Proposition bey ihnen geschehen, und ich deswegen ehemahlen auch meine gedancken eröfnet gehabt, so habe zwar nichts zu erinnern wenn die Neue Proposition aus einem andern Principio gehet, solte aber mein Principium reassumiret [= wieder aufgenommen; TG] werden, so hätte ich einig jus interventionis. Mein Principium bestund in folgenden mit wenigen ... [folgt eine komprimierte Darlegung seiner älteren Grundgedanken; TG] ... Sollte nehmlich die neüe proposition aus einem andern principio gehen, so stelle ich sie dahin, sollte man sich aber des principii so ich angewiesen bedienen wollen, daß nehmlich die resistenz des eisern seiles in die wage gebracht, und damit aufgehoben würde, es geschehe gleich solches durch ein rund umbgehendes, oder sonst vermehrtes seil oder andere dazu dienliche application, so auff viele weise zu variiren, so hätte ich billig zu interveniren. (Leibniz; AA I-Suppl., 5 f.; 1693)
\end{abstract}

Die Passage bezieht sich auch auf das Verhältnis einer (hier physikalisch unterfütterten) Grundidee („Principium“), deren Ausführung aber in der technischen Umsetzung variabel sein kann (,auff viele weise zu variiren“). Leibniz reklamiert hier den Schutz seiner wesentlichen technisch-physikalischen Grundidee, die nach seinem Verständnis auch die unterschiedlichen praktischen Ausführungs- oder Umsetzungsweisen mit einschließt. Prioritäts- und Eigentumsansprüche und die darauf bezogenen (Teil-)Kontroversen finden wir in dieser Zeit also nicht nur in der Ideen- und Wissenschaftsgeschichte, sondern auch in Anwendungsbereichen der Technik und des Ingenieurwesens.

\title{
5.3 Strategische Aspekte der Verständigung und der Auseinandersetzung in einem Verwaltungsumfeld
}

Die Kontroversen und Teilkontroversen, mit denen wir es hier zu tun haben, sind nicht Teil der gelehrt-öffentlichen Publikationsökologie der „République des Lettres“, sondern der Verwaltungskommunikation an einem Hof, bei der die Beteiligten auch als Rollenträger mit bestimmten Aufgaben und Rechten handeln, die aber in der Auseinandersetzung selbst auch Gegenstand der Verhandlung werden können. In einer solchen Arena spielen neben den wissenschaftlich-technischen auch strategische Aspekte der Verständigung und ggf. der Auseinandersetzung eine zentrale Rolle. Wir haben bei der letzten Textstelle im Abschnitt 5.2 bereits gesehen, dass Leibniz seine Anfrage an den Berghauptmann außerhalb des regulären Verwaltungsschriftwechsels anbringt und ihn bittet: „Sie belieben mir in privato einig liecht deswegen wiederfahren zu laßen [...] ohne das eben vor der hand nöthig, die Sach im Bergamt vorzutragen, und meine intervention ruchbar zu machen“ (AA I-Suppl., 6; 1693).

Die Bestandteile der Verständigung sind zum Teil schriftlich, zum Teil aber auch mündlich. Die mündlichen Anteile können wir leider nur dann rekonstru- 
ieren, wenn sie einen Niederschlag in den Quellen gefunden haben. So finden wir in den schriftlichen Unterlagen zum Beispiel strategische Erwägungen, die sich auf mündliche oder schriftliche Beiträge beziehen, die in einer bestimmten Konstellation wünschenswert sind. So schreibt etwa Leibniz an Leidenfrost, „daß er [Leidenfrost; TG] die sache [das Windmühlenprojekt; TG] bey dem Herrn Landtrosten aufs beste anbringen und auch sonst kräfftigst befördern wolle.“ (AA I-2,139). Umgekehrt schickt Leidenfrost Kommentare an Leibniz, die sich auf mögliche Einschätzungen und kritische Substanz anderer Beteiligter, z. B. der Gewerke, beziehen. Man kann hier also eine Art von kollaborativer und strategischer Behandlung von kontroverser Substanz erkennen, die auf die Interessen und Voraussetzungen der anderen Beteiligten im Feld (z. B. finanzielle Interessen der Gewerken) abgestimmt ist.

... da so viel daneben verstanden, daß es in allen Gruben unmüglich würde zu practisieren seyn, sonderlich bey denen 1.) die im Grunde zwischen den Bergen gelegen, als da der Wind seinen Effect nicht würde thun können, 2.) die Sich des Stollens gebraucheten würden sagen, daß Sie selbiges nicht bedarff, 3.) vermeinen Sie daß wen es ein universal Werk werden solte, man es erst en particulier bey einer oder andern zechen anfangen und sehen solte ... 4) dürffte es eine schwürigkeit bey denen Gewercken abgeben, absonderlich wen die Sich einem newen qvartalen Oneri unterwerffen solten, da Sie bereits den 10 und 9ten geben, auch noch mehr abführen. Jch habe alles nach vermögen beantwortet ... (Leidenfrost, AA 1-2, 142.27 ff., 1697)

Die einzelnen Schreiben beziehen sich immer wieder auch auf spezifische Aspekte der zeitlichen Dynamik, der Organisationsstruktur und der Beteiligungsrollen im Rahmen dieser komplexen Verwaltungskommunikation. Im folgenden Beispiel wird etwa die zeitliche Abfolge bestimmter Schriftsätze und auch die Frage, wie sie genau formuliert sein müssten, thematisiert:

Jch möchte wündschen daß dieses mein schreiben ante relationem expeditam [= vor der Stellungnahme des Bergamtes an den Herzog, TG] ankäme, so köndte M.h.H. dahin trachten wie einige vorträgliche dinge hinein gerückt werden möchten (...)

Ferner so bleibt diese difficultät, wenn denen gewercken freystehen soll das werck anzunehmen oder nicht, wird folgen, daß die so sich dagegen opiniatriren dabey gewinnen werden (...) Köndte also vielleicht in der Relation Serenissimo dieser Vorschlag gethan werden;

... so hielten Sie dafür; es köndte Serenissimus ein decret publiciren laßen, des ungefährlichen inhalts, „Weilen Serenissimo einige sehr scheinbare inventiones zu hebung der waßer vermittelst des windes vorbracht worden ... So hätten Jhre Durchlt vor rathsam gefunden, das werck folgender maßen zu reguliren; wie es die billigkeit, und der gewercken augenscheinlicher vortheil clärlich mit sich brächte; Nehmlich etc. (Und da müsten nun die conditiones in specie folgen.)“ ... (Leibniz; AA I-2, 144.26 ff.; 1697)

Das Beispiel zeigt auch, dass in der Verwaltungskommunikation partiell andere Beteiligungskonstellationen vorliegen als im öffentlichen Teil der Wissen- 
schaftskommunikation. Die Textdaten in der hier vorgestellten Kontroverse zeigen, dass alle Beteiligten eigene strategische Ziele verfolgen und diese Ziele mit unterschiedlichen schriftlichen oder mündlichen, offiziellen oder „privaten“ bzw. internen Beiträgen umzusetzen versuchen. Leibniz, Leidenfrost, die unterschiedlichen Funktionsträger des Bergamts, der Herzog, die Gewerke und ihre Mitglieder sind Akteure in einer Arena, in der wissenschaftliche, technische und ökonomische Gesichtspunkte auf komplexe Weise zusammenspielen und zum Teil auch konkurrieren. Es stellt sich nun die Frage, wie der „kommunikative Kosmos“ der Verständigungsmittel in dieser Art von kontroverser wissenschaftlich-technisch-ökonomischer Verwaltungskommunikation strukturiert ist.

\subsection{Leibnizens Technologie-Vorschläge, ihr „kommunikativer Kosmos" und ihr Kontroversenpotential}

Im Unterschied $\mathrm{zu}$ denjenigen wissenschaftlichen Kontroversen, die in der Frühen Neuzeit mit gedruckten und öffentlich zugänglichen Streitschriften ${ }^{7}$ ausgetragen wurden, spielten sich die Auseinandersetzungen um Leibnizens Technologie-Vorschläge in einem intern gestuften Bereich der Verwaltungskommunikation ab, der unterschiedliche „Öffentlichkeitsgrade“ im Rahmen des Verwaltungshandelns umfasste, vom persönlichen Gespräch bzw. dem persönlichen Briefwechsel bis zum Gremienschriftwechsel z.B. mit dem Bergamt oder Schreiben, die an mehrere Adressaten gingen. Zu diesen amtlichen Schriften gehörten etwa Denkschriften, in denen neue Ideen und mehr oder weniger detailliert ausgearbeitete Planungen dargestellt wurden, Gutachten, Stellungnahmen aller Art, Beschwerden, Materialbestellungen, Ausgabeanweisungen für Material und Quittungen sowie Briefe spezifischen oder gemischten Inhalts. Die herzoglichen Reskripte nehmen in diesem Bereich eine gewisse Sonderstellung ein, weil sie neue rechtsgültige Zustände herstellen können, z. B. die Anweisung zur Erprobung oder Einstellung einer neuen Technologie. Viele Züge im kommunikativen Verwaltungshandeln sind deshalb letztlich darauf gerichtet, günstige Entscheidungen der Obrigkeit herbeizuführen.

Wie schon erwähnt, sind auch Gespräche und mündliche Verhandlungen ein wichtiger Bestandteil dieses kommunikativen Bereichs. Die mündlichen Anteile sind aber nur über Berichte über Gespräche bzw. Erwähnungen ermit-

7 Brieflich ausgetragene Kontroversen hatten zunächst nur internen Charakter, sie konnten durch die Weitergabe von Briefen bzw. Briefinhalten immerhin in kleinen Gruppen nachvollzogen werden. 
telbar und ggf. auch in Grundzügen rekonstruierbar. Man muss davon ausgehen, dass es wie in heutigen Situationen strategischen Handelns auch in der Frühen Neuzeit Situationen gegeben hat, in denen es gerade darauf ankam, dass Formen der Verständigung keine kommunikativen Spuren hinterlassen. Immerhin gibt es in den edierten Materialien zum Harzbergbau immer wieder Hinweise darauf, wie mündliche und schriftliche Formen der Verständigung, der Auseinandersetzung und ggf. der strategischen Verfolgung von Zielsetzungen in der zeitlichen Dynamik und im Zusammenspiel unterschiedlicher Personen und Personengruppen verlaufen sind.

Die Tatsache, dass diese Formen der Auseinandersetzung im Rahmen der internen Verwaltungskommunikation vonstattengingen, unterscheidet sie zunächst von den gedruckt-öffentlichen Formen der wissenschaftlichen Kontroversen. Aber im Hinblick auf die Verfahren der Kontroversenführung und die Textorganisation gibt es doch auch bemerkenswerte Übereinstimmungen, denen wir uns nun zuwenden.

\subsection{Traditionelle Darstellungs-Elemente und -Verfahren}

Ich habe bisher darauf hingewiesen, dass die Auseinandersetzung um die technischen Neuerungsvorschläge Leibnizens vor allem Gegenstände der internen Verwaltungskommunikation waren. Im Hinblick auf die Verfahren und Darstellungsmittel der Kontroverse finden wir aber auch bemerkenswerte Gemeinsamkeiten mit der wissenschaftlichen Kontroversenpraxis im 17. Jahrhundert. Diese Gemeinsamkeiten kann man vor allem im Punkt-für-Punkt-Verfahren und in der textuellen Praxis einer Kombination von Wiedergabe und Erwiderung bei der Behandlung kontroverser Punkte sehen.

Für das Punkt-für-Punkt-Verfahren und seine Aspekte in der Behandlung kontroverser Teilfragen finden wir eine ganze Reihe unterschiedlicher Bezeichnungen wie z. B. articulatim, distinctè, fuß fur fuß, punct, dubium und andere (AA I-4, 68). Es handelt sich hierbei vielfach um fremdsprachige Bezeichnungen, die in einer deutschsprachigen Umgebung genutzt werden.

Auch im Hinblick auf die Art der Behandlung der einzelnen kontroversen Streitpunkte finden wir ein traditionelles textuelles Verfahren, das Verfahren, zu einzelnen „Punkten“ zunächst die gegnerische Auffassung wiederzugeben, um dann darauf zu antworten. Dieses Schema von Wiedergabe plus Beantwortung ist in der Leibniz-Kontroverse etwa in folgendem Abschnitt zu erkennen:

Was also die objection betrifft daß der wind sich in einem tage offt ändern könne, und würde bey stehendem winde das waßer aus dem teiche nicht gleich kommen können, und also die pompe die zeit über feyern, item wenn der wind wieder angienge, würde das 
einmahl im graben stehende waßer unnöthig verstreichen, darauf dienet zur antwort, daß der fall alleine sich zutragen kan wenn der wind starck und daher des waßers gar nichts gelaßen worden (...) (Leibniz an den Herzog; AA I-2, 195.20 ff.; Kursiv-Hervorh. T. G.)

Das Punkt-für-Punkt-Verfahren dient unter anderem dazu, in der Verständigung und der Auseinandersetzung die thematische Übersicht sicherzustellen, also die Übersicht darüber, welche Teilpunkte bereits angesprochen wurden, mit welcher „Gründlichkeit“ sie beantwortet wurden, welche Punkte noch nicht oder noch nicht zureichend behandelt wurden und wie man demzufolge den „Spielstand“ im Hinblick auf die kontroverse Substanz beurteilen kann. Im folgenden Textbeispiel beruft sich Leibniz zunächst auf Vorgaben des Herzogs, denen zufolge die Auseinandersetzung „ordentlich“, also entlang von geregelten Verfahren, und „distinctè“, Punkt für Punkt, erfolgen solle. Auf dieser Grundlage beklagt sich Leibniz dann, dass sich das Bergamt nicht an diese kommunikativen Verfahrensvorgaben gehalten hat. Seine Beschwerden richten sich unter anderem auf die fehlende Punkt-für-Punkt-Behandlung, auf die fehlende thematische und sequentielle Ordnung, und er schließt Überlegungen an, was mögliche strategische Ziele und Hintergründe für diese aus seiner Sicht unordentliche Vorgehensweise seiner Gegner sein könnten (,die sachen verdunckeln“). Es ist klar, dass das Bergamt in dieser Darstellung nicht nur sachlich, sondern auch im Hinblick auf die Kommunikationsmoral ein schlechtes Bild abgibt. Man sieht an diesem Beispiel erneut, dass sich „sachliche“ Argumente und Aspekte, die man traditionellerweise „ad hominem“ oder „ad personam“ klassifizieren würde, nicht immer säuberlich trennen lassen. Leibniz schreibt zu diesen Verfahrensaspekten:

5to So soll man laut des Gndsten Befehls auch ordentlich verfahren ... zu welchem ende ich ... ihre 17 dubia ... ordentlich auch distinctè beantwortet, aber anstatt einer ordentlichen replic, so auf meine antwort articulatim gerichtet gewesen wäre, vnd deroselben fuß fur fuß (wie ich ihren dubiis) gefolget hatte, übergibt man eine Schrifft unterm titul einer erklärung oder meinung, vnd observiret darin gar keine sichtbahre ordnung nicht, sondern berühret carptim vnd desultoriè balt dieses balt jenes, aber dies ist eben das rechte mittel die sachen lange aufzuhalten, vndt die Leute herumb zufuhren, daß man nach etlichen schrifftwechselungen nicht weiß wo man daran ist vnd immer im Circkel herumb gehet, hingegen meine methode ... ist das rechte mittel, die dubia balt zu exhauriren, vnd zum ende zukommen. Jch wil von des $\mathrm{H}$. concipienten intention nicht urtheilen, muß aber bekennen, daß ins gemein advocaten, so die sachen verdunckeln wollen, eß eben so machen. (Leibniz; AA I-4, 68.9 ff.; 1684)

Interessant an dieser Passage ist, dass aufgrund der Kritikpunkte an der „fehlerhaften" Kontroversenführung des Bergamts dann auch eine Art von Spielstandsbestimmung formuliert wird. Leibniz schreibt: „,... vnd bleiben also alle meine unberührte solutiones dubiorum in ihrem werth" (Leibniz; AA I-4, 68.23 ff.). Punkte, die vom Gegner entweder gar nicht oder nicht zureichend 
beantwortet wurden, verbucht Leibniz also als eigenen Gewinn. Auch diese Praxis ist nicht unüblich in den Kontroversen der Frühen Neuzeit, in den religionspolitischen Kontroversen der Frühen Neuzeit finden sich immer wieder Beispiele dafür. Leibniz gibt in dieser Passage auch eine funktionale Begründung für die strenge Befolgung des Punkt-für-Punkt-Verfahrens. Mit den Ausdrücken exhauriren bzw. zum Ende kommen bezieht er sich auf die Notwendigkeit, in solchen Auseinandersetzungen eine belastbare Entscheidung herbeizuführen, einen klaren Ausgang zu bestimmen. Hier zeigt sich wiederum ein gewisser Unterschied $\mathrm{zu}$ vielen öffentlichen wissenschaftlichen Kontroversen der Zeit, in denen das Ergebnis vielfach auch offen blieb.

\subsection{Kommunikative Maximen und Aspekte einer impliziten Kontroversentheorie}

Auch in den Texten, die zu Leibnizens Vorschlägen für technische Neuerungen im Harzbergbau überliefert sind, finden sich eine ganze Reihe von reflexiven Äußerungen zu den Spielarten und Prinzipien der Kontroversenführung. In einem Schreiben aus dem Jahr 1684, das oben schon in Auszügen zitiert wurde, klagt Leibniz über die Verletzung mehrerer kommunikativer Prinzipien (AA I-4, 66 ff.). Er beklagt fehlenden Respekt (kein glimpff). Er kritisiert die Haltung des Bergamts als autoritativè, seine Vertreter tun so, als sei schon ausgemacht, wer recht hat. Leibniz mahnt die fehlende Spezifizierung kritischer Punkte, die das Bergamt erwähnt, an (specialia; in specie). Nach Leibniz fehlt in den Ausführungen des Bergamts die argumentative Untermauerung (rationes). Er kritisiert, dass das Bergamt die ordnungsgemäße Punkt-für-Punkt-Behandlung nicht leiste und nicht articulatim und distinctè in den Schriftsätzen vorgehe. Er kritisiert die fehlende Unparteilichkeit des Bergamts bei der Beurteilung der Vorschläge. Zum Vorwurf der Unparteilichkeit gibt er darüber hinaus eine spezifische Ausführungsbestimmung, nämlich die Erwartung, dass auch ein Kritiker in der Lage sein sollte, $\mathrm{zu}$ einem strittigen Punkt etwas Konstruktives, Sachdienliches beizutragen. In die Richtung des Bergamts beklagt Leibniz sich: „daß ihnen gar nichts dienliches beyfallen solte, qvis credat?“ (,Es muss den Vertretern des Bergamts doch auch irgendetwas Konstruktives, Sachdienliches zu meinen Vorschlägen eingefallen sein. Warum sagen sie es nicht?`). In diesem Zusammenhang unterstellt Leibniz dem Bergamt eine Strategie der Schikane und der Verdunkelung, er fordert gleichzeitig eine Unterlassung solcher Haltungen in Kontroversen und auch eine Unterlassung entsprechender Handlungsweisen (Sophismata; AA I-4, 67.25 ff.)

Diese ersten Hinweise auf die Grundstrukturen einer weitläufigen Kontroverse über technische Innovationen im Bergbau, in denen wissenschaftliche, 
technisch-praktische und auch ökonomische Gesichtspunkte in einem Kontext der Verwaltungskommunikation eng verbunden sind, sollten zweierlei zeigen. Zum einen wurde deutlich, dass der Bereich der Technik und der Techniknutzung um 1700 auch eine Reihe von ganz eigenen Aspekten der Kontroversenorganisation mit sich bringt. Zum anderen sind aber die Übereinstimmungen und Gemeinsamkeiten mit der Art der Kontroversenführung in den wissenschaftlichen Kontroversen der Zeit auch mehr als deutlich. Es wird eine lohnende Aufgabe sein, die Praxis der Kontroversenführung und auch die reflexiven Textstellen, die eine Rekonstruktion einer impliziten Kontroversentheorie erlauben, auf die editorisch bereits gut dokumentierte und kommentierte Kontroversenlehre und -praxis von Gottfried Wilhelm Leibniz zu beziehen, wie sie vor allem in den Arbeiten von Marcelo Dascal vorgelegt wurde (Dascal 2006; 2010).

\section{Die deutsche Phlogiston-Kontroverse am Ende des 18. Jahrhunderts: Zeitschriften und die Prinzipien der neuen Chemie}

Gegen Ende des 18. Jahrhunderts diskutierten deutsche Chemiker die neuen Thesen von Lavoisier über die Verbrennung, die bereits früher in Frankreich zu heftigen Auseinandersetzungen geführt hatten (vgl. Frercks 2008; Seils 1995). Lavoisier hatte im Kern die noch heute gültige Sauerstoff-Theorie der Verbrennung entdeckt, die ältere Phlogiston-Theorie nahm einen eigenen Stoff an, das Phlogiston, das bei Verbrennungs- und Reduktionsprozessen abgegeben bzw. aufgenommen wurde. Die deutschsprachige Kontroverse um Phlogiston und Sauerstoff weist eine Reihe von interessanten Eigenschaften auf, unter anderem eine „Bekehrung“, was in wissenschaftlichen Kontroversen der Frühen Neuzeit eine sehr seltene Erscheinung ist.

In kommunikativer Hinsicht ist neu, dass die Kontroverse in wissenschaftlichen Zeitschriften stattfindet, die im 18. Jahrhundert neu gegründet wurden, z. B. das von Gren herausgegebene „Journal der Physik“, dessen erster Jahrgang 1790 erschien. Mit der Hinwendung zu den Zeitschriften ist auch eine Abkehr vom traditionellen Streitschriften-Modell verbunden. Dies führt zum einen zu einer starken Beschleunigung in der zeitlichen Dynamik der Kontroverse, zum anderen zu einer Abkehr vom Prinzip der vollständigen Widerlegung. Aufgrund des geringeren Umfangs von Kontroversenbeiträgen sind die Autoren gezwungen, eine Auswahl von Punkten und damit eine Relevanzbewertung vorzunehmen. Punkte, die nicht bearbeitet werden, gelten in die- 
sem Zusammenhang nicht als „verloren“, wie es im älteren Streitschriften-Modell der Fall gewesen wäre.

Hinzu kommt, dass die Kontroverse starke intermediale Zusammenhänge aufweist: So werden unter anderem Auszüge aus internationalen Zeitschriften, die sich auf den Streitgegenstand beziehen, übersetzt und es werden Briefe entweder ganz oder in Auszügen als Stellungnahmen zu einzelnen Punkten abgedruckt. Auch hier sind internationale Verflechtungen unter den Chemikern erkennbar.

Die im 18. Jahrhundert als moderne Wissenschaft neu begründete Chemie hatte einen starken experimentalwissenschaftlichen Charakter. Damit ging auch eine Veränderung im Repertoire der kommunikativen Züge und Darstellungsformen einher. Dazu gehören unter anderem solche mehr oder weniger komplexen sprachlichen Handlungen wie „eine Versuchsanordnung beschreiben“, „erklären, wie eine Versuchsanordnung mit einem Streitpunkt zusammenhängt“, „Ergebnisse eines Experiments beschreiben und Schlussfolgerungen für einen Streitpunkt formulieren“, „eine Versuchsanordnung (als untauglich) kritisieren“ und andere.

Auch die Chemiker, die an der deutschsprachigen Phlogiston-Kontroverse beteiligt waren, berufen sich vielfach auf kommunikative Maximen. Im Vergleich zu älteren Kontroversen ist besonders ein Prinzip der Höflichkeit und des respektvollen Umgangs sowie ein Prinzip der unvoreingenommenen Wahrheitssuche zu erkennen. Dieses Prinzip führte dazu, dass die zentrale Persönlichkeit des phlogistischen Lagers, Gren, eine Art von schrittweiser „Bekehrung “ erfuhr, die ihn schließlich dazu brachte, die Auffassungen von Lavoisier $\mathrm{zu}$ akzeptieren und dies auch in seinen Schriften $\mathrm{zu}$ bekennen. Grens Bekehrung rief nicht geringes Aufsehen in der Fachwelt hervor. So schreibt Leopold von Buch an Johann Carl Freiesleben:

Kan ich Ihnen wohl eine interessantere und wichtigere Nachricht erzählen, als die, die mir selbst noch ganz unglaublich vorkomt, so gewiß sie auch ist. - Gren ist ein Antiphlogisticker geworden!! Und das recht ernstlich; er hat es mir auf eine Art erzählt, die ihm unendlich viel Ehre macht. (zit. nach Seils 1995: 7)

Da in diesem Brief der Sensationscharakter dieser Neuigkeit auch im Schriftbild gekennzeichnet wird, folgt nun noch eine Abbildung der Brief-Passage:

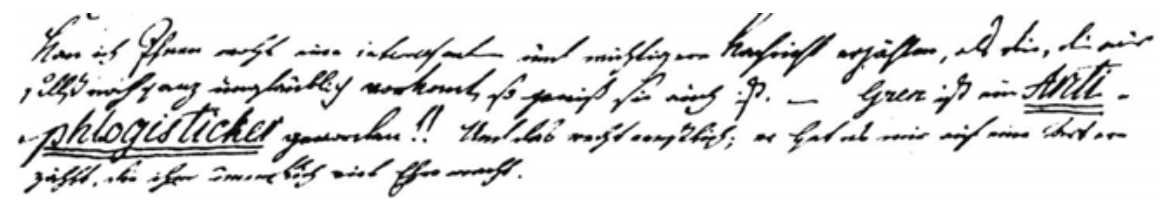

Abb. 3: Briefauszug Leopold von Buch über die „Bekehrung“von F. A. C. Gren; Quelle: Seils 1995. 
Diese Kontroverse, die ausführlicher in einer Studie im Band von Fritz \& Gloning (to appear) analysiert und kommunikationsgeschichtlich eingeordnet wird, ist vor allem in dreierlei Hinsicht bemerkenswert. Sie zeigt zum einen, wie ein mediengeschichtlicher Umbruch, die Hinwendung zur Nutzung von Zeitschriften, auch die kommunikative Praxis der Kontroversenführung nachhaltig veränderte. Sie zeigt darüber hinaus, wie auch die Anforderungen einer bestimmten Wissenschaftsdisziplin das Repertoire der Handlungsformen in Kontroversen prägen kann, hier vor allem die Erfordernisse in Bezug auf Experimente und ihren Bezug zu einzelnen Streitpunkten. Schließlich und drittens ist in dieser Kontroverse auch zu erkennen, wie allgemeine ideen- und kulturgeschichtliche Tendenzen der Aufklärung, z. B. Höflichkeit und Wahrheit, sich auch in den Kontroversenidealen und den impliziten Theorien der Kontroverse niederschlagen.

\section{Ergebnisse, Ausblicke, Forschungsperspektiven}

Die hier vorgestellten Kontroversen aus dem 17. und 18. Jahrhundert sollten zunächst zeigen, dass Kontroversen eine wichtige Rolle für die gelehrte Verständigung und die Wissenschaftskommunikation in den unterschiedlichen Feldern und $\mathrm{zu}$ unterschiedlichen Zeiten spielten. ${ }^{8}$ Sie zeigen weiterhin, dass Streitschriften ein zentrales Kommunikationsmittel in Kontroversen sind, die typischerweise eine Punkt-für-Punkt-Zerlegung des Streitgegenstandes aufweisen und die einzelnen Punkte mit einem Wiedergabe-plus-Antwort-Schema organisieren. Dieses Streitschriften-Modell erlaubt zum einen eine systematische Themenorganisation, sie erlaubt zum anderen die „Buchführung“ über den jeweiligen Stand der Kontroverse, sie organisiert darüber hinaus die intertextuellen Bezüge zwischen den Streitschriften. Um den Einschränkungen dieser Art der thematischen Organisation zu entgehen, nutzen Autoren immer wieder Exkurse und Digressionen, die es ihnen erlauben, sich von den gegnerischen Vorgaben $\mathrm{zu}$ lösen und eine eigene Agenda zu verfolgen.

Das Streitschriften-Modell weist über die fachlichen Zonen hinweg wesentliche strukturelle Gemeinsamkeiten und tendenziell nur wenige Unterschiede in verschiedenen Wissenschaftsbereichen auf. So finden wir wesentliche Übereinstimmungen in wissenschaftlichen, theologisch-religionspolitischen und in

8 Weiterführend: Bremer 2005; Bremer \& Spoerhase 2015; Dascal 1998; 2010; Fritz 2003; 2005a, b; 2008; 2010; 2012; 2013; 2016a, b; Gloning 1999; 2005; 2013. Siehe demnächst auch die Studien in Fritz \& Gloning (to appear). 
technischen bzw. auf wirtschaftliche Anwendungen ausgerichteten Kontroversen.

Im historischen Längsschnitt war es vor allem der mediale Umbruch, der mit der Nutzung von Zeitschriften verbunden war, der auch einschneidende Folgen für die kommunikative Praxis der wissenschaftlichen Kontroversenführung hatte. Vergleichbare Umbrüche haben wir in den letzten beiden Jahrzehnten durch die zunehmende Digitalisierung der Wissenschaftskommunikation beobachtet (vgl. Gloning \& Fritz 2011; Fritz \& Gloning 2012).

Die Untersuchung von historischen Wissenschaftskontroversen ist aber nicht nur ein Beitrag zur Erforschung der Geschichte von Gelehrtensprachen und der historischen Wissenschaftskommunikation, sondern auch ein interessantes Testgelände für die Sprachwandelforschung und die Theoriebildung im Bereich der Historischen Pragmatik, insbesondere einer Theorie der Geschichte von Kommunikationsformen (vgl. Keller 1990; Fritz 1994; 1995; 2012; Beckmann \& König 1995; Luckmann 1986).

Kontroversen sind in vielen gelehrten Biographien ein nicht unerheblicher Bestandteil im Haushalt der Lebenszeit und der wissenschaftlichen Aktivitäten, z. B. bei intellektuellen Schwergewichten wie Kepler oder Leibniz. Die Untersuchung von Kontroversen trägt in solchen Fällen auch dazu bei, die individuellen Kommunikationsprofile und die je eigene Prägung des Sprachgebrauchs einzelner Wissenschaftler oder bestimmter Gruppen von Gelehrten zu charakterisieren.

\section{Quellen und Literatur}

\section{Quellen}

Feselius, Philipp (1609): Gründtlicher Discurs von der Astrologia Judiciaria (...). Straßburg. Gehema, Janus Abraham à (1689): Die beste Zeit-Vertreib. Bremen.

Gehema, Janus A. à (1688): Grausame Medicinische Mord-Mittel/ Aderlasse/ Schröpffen/ Purgiren/ Clistiren/ Juleppen/ und Ohnmacht-machende Hertzstärckungen (...). Bremen. Nachdruck Lindau 1980.

Geuder, Melchior F. (1689): Heilsame Medicinische Lebens-Mittel/ Denen grausamen Medicinischen Mord-Mitteln/ Herrn D. Jan. Abrah. à Gehema Entgegen gesetzt. (...) Ulm. Nachdruck Lindau 1982.

Kepler, Johannes (1938): De stella nova in pede serpentarii (1606). In: Johannes Kepler, Gesammelte Werke. Band 1. Hrsg. von M. Caspar. München, 147-356 und 441-487.

Kepler, Johannes (1941): Gesammelte Werke. Band 4: Kleinere Schriften 1602/1611. Dioptrice. Hg. von M. Caspar und F. Hammer. München.

Kepler, Johannes (1941): Tertivs interveniens. Das ist/ Warnung an etliche Theologos, Medicos vnd Philosophos (...). Frankfurt a.M. (G. Tampach) 1610. In: Johannes Kepler, Gesammelte Werke. Band 4. Hrsg. von M. Caspar und F. Hammer. München, 147-258. 
Kepler, Johannes (1941): Antwort Joannis Keppleri (...) Auff D. Helisæi Röslini (...) Discurs Von heutiger zeit beschaffenheit (...). Prag (Sesse) 1609. In: Johannes Kepler, Gesammelte Werke. Band 4. Hrsg. von M. Caspar und F. Hammer. München, 101-144.

Kepler, Johannes (1990): Gesammelte Werke. Band 12: Theologica, Hexenprozeß, TacitusÜbersetzung, Gedichte. Bearbeitet von J. Hübner, H. Grössing, F. Boockmann, F. Seck. Redaktion V. Bialas. München.

Leibniz, Gottfried Wilhelm (1927): Sämtliche Schriften und Briefe. Band I-2: Allgemeiner historischer und politischer Schriftwechsel 1676-1679. Darmstadt: Otto Reichl Verlag. [= AA I-2]

Leibniz, Gottfried Wilhelm (1950): Sämtliche Schriften und Briefe. Band I-4: Allgemeiner, politischer und historischer Briefwechsel 1684-1687. Hrsg. von der Deutschen Akademie der Wissenschaften zu Berlin. Berlin, Leipzig. [= AA I-4]

Leibniz, Gottfried Wilhelm (1991): Sämtliche Schriften und Briefe. Erste Reihe. Allgemeiner politischer und historischer Briefwechsel. Supplementband Harzbergbau. Berlin: Akademie Verlag. [= AA I-Suppl.]

Röslin, Helisäus (1597): Tractatvs Meteorastrologiphysicus. Das ist/ Auß richtigem lauff der Cometen/ zusammenleuchtung der Planeten/ etlicher Herrn Natiuiteten/ Natürliche Vermu:otungen vnd ein Weissagung. Straßburg: Jobins Erben.

Röslin, Helisäus (1605): IVDICIVM, Oder Bedencken Vom Newen Stern/ welcher den zweiten Octobris erschinen/ vnd zum erstenmal gesehen worden. Straßburg: Anton Bertram.

Röslin, Helisäus (1609): Historischer/ Politischer vnd Astronomischer naturlicher Discurs von heutiger zeit Beschaffenheit/ Wesen vnd Standt der Christenheit/ vnd wie es ins künfftig in derselben ergehn werde (...). Straßburg: Conrad Scher/ Paul Ledertz.

Röslin, Helisäus (1611): Mitternächtige Schiffarth/ VOn den Herrn Staden inn Niderlanden vor XV. Jaren vergebenlich fürgenommen/ wie dieselbige anzustellen/ daß man daselbst herumb in Orient vnd Chinam kommen möge (...). Oppenheim: H. Gallart, J. Th. de Bry.

Schaerer, Melchior (o. J.; Vorrede 1608): Prognosticon, Oder PRactica (...) Auff das Jahr/ nach der Gnadenreichen Geburt vnsers Heylands Jesu Christi M.DCIX. Nürnberg: Fuhrmann.

Schaerer, Melchior (1611): Verantwortung vnd Rettung der ARgumenten vnd Vrsachen/ welche M. Melchior Scherer/ in den Vorreden seiner zweyen Prognosticorum verschiener 1608. vnd 1609. Jahren (...) eingeführet: Wider (...) Philippum Feselium (...). 0. 0.: Böhem, Fuhrmann.

\section{Forschungsliteratur}

Beckmann, Susanne \& Peter-Paul König (1995): Wie ein Textmuster entsteht ... In: Grazer Linguistische Studien 44, 1-14.

Bremer, Kai (2005): Religionsstreitigkeiten. Volkssprachliche Kontroversen zwischen altgläubigen und evangelischen Theologen im 16. Jahrhundert. Tübingen: Max Niemeyer.

Bremer, Kai \& Carlos Spoerhase (Hrsg.) (2015): „Theologisch-polemische Sachen“. Gelehrte Polemik im 18. Jahrhundert. Frankfurt a. M.: Vittorio Klostermann.

Dascal, Marcelo (1998): The study of controversies and the theory and history of science. In: Science in Context 11, 147-154.

Dascal, Marcelo (ed.) (2006): Gottfried Wilhelm Leibniz, The Art of Controversies. Translated and edited, with an introductory essay and notes by Marcelo Dascal with Quintín Racionera and Adelino Cardoso. Dordrecht: Springer.

Dascal, Marcelo (ed.) (2010): The practice of reason. Leibniz and his Controversies (Controversies 7). Amsterdam, Philadelphia: Benjamins. 
Frercks, Jan (2008): Kommentar. In: Antoine Laurent Lavoisier, System der antiphlogistischen Chemie. Aus dem Französischen von Sigismund Friedrich Hermbstaedt. Übersetzung durchgesehen von Jan Frercks. Kommentar von Jan Frercks. Frankfurt a. M.: Suhrkamp, 181-411.

Fritz, Gerd (1994): Geschichte von Dialogformen. In: Gerd Fritz \& Franz Hundsnurscher (Hrsg.), Handbuch der Dialoganalyse. Tübingen, 545-562.

Fritz, Gerd (1995): Topics in the history of dialogue forms. In: Andreas Jucker (ed.), Historical pragmatics. Amsterdam, Philadelphia: Benjamins, 469-498.

Fritz, Gerd (2003): Dialogical structures in 17th century controversies. In: M. Bondi \& Edda Weigand (Hrsg.): Dialogue Analysis 2000. Tübingen, 199-208.

Fritz, Gerd (2005a): On answering accusations in controversies. In: Studies in Communication Sciences. Special Issue Argumentation in Dialogic Interaction. Lugano: Università della Svizzera Italiana, 151-162.

Fritz, Gerd (2005b): First person singular in 17th century controversies. In: Pierluigi Barrotta \& Marcelo Dascal (Hrsg.), Controversies and subjectivity. Amsterdam, Philadelphia, 235-250.

Fritz, Gerd (2008): Communication principles in controversies. A historical perspective. In: Frans H. Van Eemeren \& Bart Garssen (Hrsg.), Controversy and Confrontation. Relating Controversy Analysis with Argumentation Theory. Amsterdam, Philadelphia: Benjamins, 109-124.

Fritz, Gerd (2010): Controversies. In: Andreas Jucker \& Irma Taavitsainen (Hrsg.), Historical Pragmatics. Handbook of Pragmatics, Vol. 8. Berlin, New York: de Gruyter, 451-481.

Fritz, Gerd (2012): Kontroversen - Ein Paradigma für die Geschichte von Kommunikationsformen. In: Peter Ernst (Hrsg.), Historische Pragmatik. Berlin, Boston: de Gruyter, 105126.

Fritz, Gerd (2016a): Zum Sprachgebrauch wissenschaftlicher Kontroversen im Deutschen um 1600. In: Gerd Fritz, Beiträge zur Texttheorie und Diskursanalyse. Gießen: Gießener Elektronische Bibliothek, 153-171. http://geb.uni-giessen.de/geb/volltexte/2016/12024/

Fritz, Gerd (2016b): Zum Sprachgebrauch wissenschaftlicher Kontroversen im Deutschen um 1700. In: Gerd Fritz, Beiträge zur Texttheorie und Diskursanalyse. Gießen: Gießener Elektronische Bibliothek, 173-223. http://geb.uni-giessen.de/geb/volltexte/2016/ $12024 /$

Fritz, Gerd (2017): Dynamische Texttheorie. Zweite Auflage. Gießen: Gießener Elektronische Bibliothek. http://geb.uni-giessen.de/geb/volltexte/2017/12601/

Fritz, Gerd \& Thomas Gloning (2012): Critique and controversy in digital scholarly communication. New formats and their affordances. In: Frans H. Van Eemeren \& Bart Garssen (Hrsg.), Exploring Argumentative Contexts. Amsterdam, Philadelphia: Benjamins, 213-231.

Fritz, Gerd \& Thomas Gloning (Hrsg.): Historical Pragmatics of Controversies. Case Studies from 1600 to 1800. Amsterdam: Benjamins (to appear).

Gindhart, Marion \& Ursula Kundert (Hrsg.) (2010): Disputatio 1200-1800. Form, Funktion und Wirkung eines Leitmediums universitärer Wissenskultur. Berlin: de Gruyter.

Gindhart, Marion, Hanspeter Marti \& Robert Seidel (Hrsg.) (2016): Frühneuzeitliche Disputationen. Polyvalente Produktionsapparate gelehrten Wissens. Berlin, New York: de Gruyter.

Gloning, Thomas (1999): The pragmatic form of religious controversies around 1600. A case study in the Osiander vs. Scherer \& Rosenbusch controversy. In: Andreas H. Jucker, 
Gerd Fritz \& Franz Lebsanft (Hrsg.): Historical dialogue analysis. Amsterdam, Philadelphia, 81-110.

Gloning, Thomas (2002): Zur sprachlichen Form der Kepler/Röslin/Feselius-Kontroverse über Astrologie und Kosmologie um 1600. In: Marcelo Dascal, Gerd Fritz, Thomas Gloning \& Yaron Senderowicz (Hrsg.): Scientific controversies and theories of controversy (GermanIsraeli Foundation, Technical Report 3). Giessen, 35-85.

Gloning, Thomas (2005): Early Modern controversies and theories of controversy. The rules of the game and the role of the persons. In: Pierluigi Barrotta \& Marcelo Dascal (Hrsg.): Constroversies and subjectivity. Amsterdam: Benjamins, 263-281.

Gloning, Thomas (2013): August Hermann Franckes frühe Streitschriften 1689-1694. Kommunikationspraxis, Textgestalt, Wortgebrauch. In: Fest-Platte für Gerd Fritz. Hrsg. und betreut von Iris Bons, Thomas Gloning und Dennis Kaltwasser. Gießen 24. 05. 2013. http://www.festschrift-gerd-fritz.de/files/gloning_2013_franckes-fruehestreitschriften.pdf

Gloning, Thomas \& Gerd Fritz (Hrsg.) (2011): Digitale Wissenschaftskommunikation. Formate und ihre Nutzung. Gießen: Gießener elektronische Bibliothek. http://geb.uni-giessen.de/ geb/volltexte/2011/8227

Gloning, Thomas \& Jan Lüsing (2002): Die Kontroverse zwischen J. A. Gehema und M. F. Geuder (1688/1689). In: Marcelo Dascal, Gerd Fritz, Thomas Gloning \& Yaron Senderowicz (Hrsg.): Scientific controversies and theories of controversy (German-Israeli Foundation, Technical report 3). Giessen, 87-143.

Keller, Rudi (1990): Sprachwandel. Von der unsichtbaren Hand in der Sprache. Tübingen: Francke.

Luckmann, Thomas (1986): Grundformen der gesellschaftlichen Vermittlung des Wissens: Kommunikative Gattungen. In: Friedhelm Neidhardt u. a. (Hrsg.), Kultur und Gesellschaft. Opladen: Westdeutscher Verlag, 191-211.

Sdzuj, Reimund B., Robert Seidel \& Bernd Zegowitz (Hrsg.) (2012): Dichtung Gelehrsamkeit - Disputationskultur. Festschrift für Hanspeter Marti zum 65. Geburtstag. Wien: Böhlau.

Seils, Markus (1995): Friedrich Albrecht Carl Gren in seiner Zeit 1760-1798. Spekulant oder Selbstdenker? (Heidelberger Schriften zur Pharmazie- und Naturwissenschaftsgeschichte 14). Stuttgart: Wissenschaftliche Verlagsgesellschaft. 